\title{
ARTICLE OPEN SARS-CoV-2-triggered mast cell rapid degranulation induces alveolar epithelial inflammation and lung injury
}

Meng-Li Wu' ${ }^{1}$, Feng-Liang Liu ${ }^{2}{ }^{2}$, Jing Sun ${ }^{3}$, Xin $\mathrm{Li}^{4}$, Xiao-Yan He ${ }^{2,5}$, Hong-Yi Zheng ${ }^{2}$, Yan-Heng Zhou ${ }^{4}$, Qihong Yan ${ }^{4}$, Ling Chen ${ }^{4}$, Guo-Ying $\mathrm{Yu}^{1}$, Junbiao Chang ${ }^{1}$, Xia Jin ${ }^{6}$, Jincun Zhao $\mathbb{D}^{3}$, Xin-Wen Chen ${ }^{4,5}$, Yong-Tang Zheng $\mathbb{D}^{2,5 \bowtie}$ and Jian-Hua Wang ${ }^{4,7 凶}$

SARS-CoV-2 infection-induced hyper-inflammation links to the acute lung injury and COVID-19 severity. Identifying the primary mediators that initiate the uncontrolled hypercytokinemia is essential for treatments. Mast cells (MCs) are strategically located at the mucosa and beneficially or detrimentally regulate immune inflammations. In this study, we showed that SARS-CoV-2-triggered MC degranulation initiated alveolar epithelial inflammation and lung injury. SARS-CoV-2 challenge induced MC degranulation in ACE-2 humanized mice and rhesus macaques, and a rapid MC degranulation could be recapitulated with Spike-RBD binding to ACE2 in cells; MC degranulation altered various signaling pathways in alveolar epithelial cells, particularly, the induction of proinflammatory factors and consequential disruption of tight junctions. Importantly, the administration of clinical MC stabilizers for blocking degranulation dampened SARS-CoV-2-induced production of pro-inflammatory factors and prevented lung injury. These findings uncover a novel mechanism for SARS-CoV-2 initiating lung inflammation, and suggest an off-label use of MC stabilizer as immunomodulators for COVID-19 treatments.

Signal Transduction and Targeted Therapy (2021)6:428

; https://doi.org/10.1038/s41392-021-00849-0

\section{INTRODUCTION}

The Coronavirus disease 2019 (COVID-19) caused by severe acute respiratory syndrome coronavirus 2 (SARS-CoV-2) has become a global pandemic. A higher incidence of severity and deaths in older individuals has been observed. ${ }^{1}$ To curtail this disease, the nucleotide analogue, Remdesivir (Veklury), has been approved by U.S Food and Drug Administration (FDA) for treating COVID-19 in adults and older chilren (more than 12 years of age) ${ }_{i}^{2}$ another ribonucleoside analogue, molnupiravir (EIDD-2801, MK-4482), developed by Merck and Ridgeback, was recently approved by United Kingdom for oral treatment of COVID-19.3 ${ }^{3,4}$ Besides, US FDA has approved and authorized two mRNA vaccines (Pfizer-BioNTech, Moderna) and one recombinant adenovirus-26 vaccine (Janssen, Ad26.COV2.S) for population use, ${ }^{5}$ and globally more than 100 candidate SARS-CoV-2 vaccines are under development. ${ }^{6}$

A key pathologic feature of COVID-19 is the hyper-inflammatory response (also termed hypercytokinemia or "cytokine storm") in association with severe COVID-19 disease, and those inflammatory cytokines and chemokines produced in vivo lead to damage to the alveolar epithelial cells and capillary endothelial cells. ${ }^{7-15}$ Known inflammatory cytokines and chemokines, which are excessively produced in patients with severe COVID-19, include IL-6, IL-8, IL-1 $\beta$, TNF- $\alpha$, IFN- $\gamma$, MIP1 $\alpha$ and $1 \beta, C C L 2, C C L 5, C C L 20$, CXCL1, CXCL2, CXCL8, CXCL10 and CXCL17. $9,13-20$ Among them, elevated IL-6, TNF- $a$ and C-reaction protein (CRP) levels have been shown to be independent risk factors for the severity of COVID-19 disease. ${ }^{13,17,20-22}$

Therefore, targeting the hyper-inflammation loop to develop the immunomodulatory agents is an attractive strategy for developing treatment for severe COVID-19 cases. ${ }^{7,23,24}$ Some drug candidates have been tried in order to mitigate hyperinflammation and improve clinical outcomes. These include humanized anti-IL-6 receptor antibody Tocilizumab, ${ }^{25}$ anti-IFN- $\gamma$ monoclonal antibody Emapalumab, IL-1 receptor antagonist Anakinra (NCT04324021), JAK1 and JAK2 inhibitors Baricite and Rituxolitinib $^{26}$ (NCT04338958), and the Corticosteroids such as Dexamethasone. ${ }^{27-29}$ However, Tocilizumab was not effective for preventing intubation or death in moderately ill hospitalized patients; ${ }^{30,31}$ another IL- 6 receptor monoclonal antibodies Sarilumab not only failed to improve clinic outcomes and reduce mortality, but led to serious complications. ${ }^{32}$ Of note, despite the probable benefit of calming the cytokine storm, suppressing inflammation and immune response may impede on viral clearance. $^{33}$ Indeed, corticosteroid treatment suppresses the overall immune responses, but also impairs the induction of anti-viral type-I interferon responses. ${ }^{34,35}$ Therefore, the development of effective immunomodulatory agents that suppress inflammation, without compromising host immune protection, is required.

Lung is the primary target for the corona viruses. The alveoli of the lung are covered with angiotensin-converting enzyme 2

\footnotetext{
${ }^{1}$ College of Life Science, Henan Normal University, Xinxiang 453007, China; ${ }^{2}$ Key Laboratory of Animal Models and Human Disease Mechanisms of the Chinese Academy of Sciences, Kunming Institute of Zoology, Chinese Academy of Sciences, Kunming 650223, China; ${ }^{3}$ State Key Laboratory of Respiratory Disease, National Clinical Research Center for Respiratory Disease, Guangzhou Institute of Respiratory Health, The First Affiliated Hospital of Guangzhou Medical University, Guangzhou, Guangdong 510182, China; ${ }^{4}$ Guangzhou Institutes of Biomedicine and Health, Chinese Academy of Sciences, Guangzhou 510530, China; ${ }^{5}$ Bioland Laboratory, Guangzhou Regenerative Medicine and Health

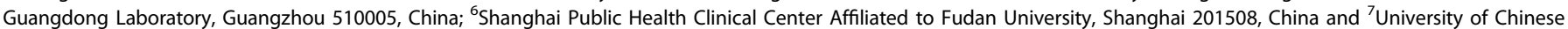
Academy of Sciences, Beijing 100039, China

Correspondence: Xin-Wen Chen (Chen_xinwen@gibh.ac.cn) or Yong-Tang Zheng (zhengyt@mail.kiz.ac.cn) or Jian-Hua Wang (wang_jianhua@gibh.ac.cn)
}

Received: 28 August 2021 Revised: 15 November 2021 Accepted: 2 December 2021

Published online: 17 December 2021 
(ACE2)-expressing epithelial cells. It is reasonable to hypothesis that the alveoli epithelial inflammation is a prerequisite for damage of both alveoli epithelial cells and capillary endothelial cells during SARS-CoV-2 infection, ${ }^{36-38}$ during which infected epithelial cells recruit and activate monocytes and macrophages to secrete pro-inflammatory cytokines, which further recruits neutrophils and activates T-cells to exacerbate inflammation. ${ }^{12}$ As shown in the postmortem lung biopsies, there were diffuses alveolar damage with necrosis of alveolar lining cells, pneumocyte type 2 hyperplasia, and linear intra-alveolar fibrin deposition, widespread vascular thrombosis with microangiopathy and occlusion of alveolar capillaries. ${ }^{39,40}$ Therefore, uncovering the mechanisms of epithelial inflammation caused SARS-CoV-2 is of uppermost priority.

MCs are tissue resident cells that strategically placed throughout the host-environment interface including the whole respiratory tract and the nasal cavity. Besides being as the main effector cells in allergy, MCs can interact with various immune cells through release of soluble factors or direct contact to beneficially or detrimentally regulate immune inflammations. ${ }^{41-43} \mathrm{~A}$ variety of pathogens including DNA/RNA virus, fungi, bacteria or their products can activate MCs, and induce secretion of cytokines and chemokines through degranulation-dependent or degranulationindependent pathways. ${ }^{43}$ The released mediators can drive the recruitments of imune effector cells for pathogens clearance; alternatively, these inflammatory mediators may induce inappropriate inflammatory responses to disrupt the epithelialendothelia barriers for promoting pathogens invasion. ${ }^{43}$ In SARSCoV-2 infection, the postmortem lung biopsies of COVID-19 patients show a massively increased density of perivascular and septal MCs, suggesting MCs were recruited to the alveolar septa to play certain unknown functions. ${ }^{44,45}$ SARS-CoV-2 infection triggered $M C$ infiltration into the pulmonary parenchyma of african green monkeys. ${ }^{46}$ These evidences indicate a potential role of MCs in SARS-CoV-2 pathogenesis.

Therefore, in this study, we investigated the role of MCs in SARS-CoV-2 infection, and examined whether SARS-CoV-2 could induce $M C$ degranulation and its sequential role in virus-induced alveolar epithelial inflammation and lung injury, and we hope to find the potent immunomodulators to treat COVID-19.

\section{RESULTS}

SARS-CoV-2 induces MC degranulation in lung of ACE2-humanized mice

To investigate whether SARS-CoV-2 could induce MC degranulation in vivo, the ACE2-humanized inbred mice, ${ }^{47}$ termed C57BL/ $6 \mathrm{~N}$-Ace $2^{\text {em2(hACE2-WPRE,pgk-puro)/CCLA }}$, were intranasally infected with SARS-CoV-2 (strain 107) at a dose of $2 \times 10^{6} \mathrm{TCID}_{50}(50 \%$ tissue culture infective dose), then euthanized at the different days postinfection (dpi) to harvest lung tissues for histological analysis. The same amount of PBS was inoculated as the mock infection. Our results confirmed previous observation that SARS-CoV-2 virions were mainly distributed in the peri-bronchus and bronchioalveolar-duct junction, ${ }^{47-49}$ as shown by immunostaining of SARS-CoV-2 nucleocapsid protein at 1- and 3-dpi (Supplementary Fig. 1). MCs and their degranulation are indicated by the metachromatic labeling with Toluidine blue (T. blue). ${ }^{50}$ Compared with mock-infection (Fig.1a), at the $1 \mathrm{dpi}$, the accumulation of MCs in peri-bronchus was observed and the release of granules was found in the alveolar space (Fig. 1c). The MC degranulation over the course of SARS-CoV-2 infection could be seen with abundance of released granules distributed widely in alveolar spaces (Fig. 1C, e, g). The lung lesions around the area of MC accumulation and degranulation were examined by staining with Hematoxylin and eosin (H.E.). Compared with mock-infection control (Fig. 1b), lung lesions, including inflammatory cells (lymphocytes and monocytes) infiltration, hemorrhage, alveolar septal thickening, and mucosa desquamation, were observed around the areas of $M C$ accumulation and degranulation (Fig. 1d, f, h). The pathological score was assessed according to the degree of lung tissue lesions and MC count in lung sections was calculated (Fig. 1i).

To ensure the above observations are not limited to one experimental model of SARS-CoV-2 infection, another mouse model, namely Ad5-hACE2-transduced BALB/c mice, ${ }^{51}$ were used. The mice were intranasal challenge with $7 \times 10^{4} \mathrm{TCID}_{50}$ SARS-CoV-2, then sacrificed at different times to harvest the lungs for histological staining. Similar results were obtained. Compared with the mock-infection (Supplementary Fig. 2a), SARS-CoV-2 challenge induced a MC degranulation in the peribronchus and bronchioalveolar-duct junction (Supplementary Fig. 2c, e, g). Simultaneous H.E. staining of the adjacent lung section showed lung lesions around these areas (Supplementary Fig. $2 d, f, h$ ), but not in mock-infected mice (Supplementary Fig. $2 b)$. The pathological score was assessed according to the degree of lung tissue lesions and MC count in lung sections was calculated (Supplementary Fig. 2i). Taken together, these data demonstrate convincingly that SARS-CoV-2 infection induces MC accumulation and degranulation around the area of lung lesion in ACE2-humanized mice.

SARS-CoV-2 induces MC degranulation in lung of rhesus macaque Having firmly established a pathogenesis model in mice, we went on the investigation whether it was producible in nonhuman primate, which has often been considered as the translational gate-keeper before any scientific discovery is progressed to human testing. The SARS-CoV-2 can infect Chinese rhesus macaques (chRMs) (Macaca mulatta), in which much of the characteristics of immunological pathogenesis of human COVID19 can be recapitulated; notably, aged chRMs showed the delayed but more severe cytokine storm and higher immune cell infiltration, compared with young chRMs. ${ }^{52}$ This model is therefore most suited for in-depth dissection of inflammatory responses in relation to $M C$ activation.

We confirmed that SARS-CoV-2 infection led to MC degranulation in lungs of chRMs. The young chRMs (3- to 6-year old) (Fig. 2) or aged chRMs (17- to 19-year old) (Fig. 3) were anesthetized, then intratracheally infected with SARS-CoV-2 $\left(1 \times 10^{7} \mathrm{TCID}_{50}\right)$ (strain 107). The animals were sacrificed at 7 or $15 \mathrm{dpi}$, and lungs were harvested and sectioned for T. blue staining to examine changes in MCs. Compared with the mockinfections (Fig. 2a, 3a), SARS-CoV-2 challenge recruited MCs accumulation in the peri-bronchus and bronchioalveolar-duct junction at both 7- and 15- dpi, with noted MC degranulation (Figs. 2b-f, 3b-d). As expected, compared with young chRMs (Fig. 2b-f), aged chRMs have showed more extensive SARS-CoV2-induced MCs accumulation and degranulation (Fig. 3b-d). Taken together, we demonstrate that SARS-CoV-2 induces MC degranulation in lung of rhesus macaque.

The binding of Spike-RBD to ACE2 triggers a rapid MC degranulation

To further investigate the molecular mechanism responsible for SARS-CoV-2-caused MC degranulation, we used human MC cell line LAD2 cells and first examined whether SARS-CoV-2 could induce LAD2 cell degranulation. MC degranulation can be assessed by the intensity reduction of immunostaining of cytoplasmic avidin granules as previously described. ${ }^{50,53}$ The cells were treated with SARS-CoV-2 (M.O.I.=1) (strain 2019-nCoV WIV04), ${ }^{54}$ and results displayed that SARS-CoV-2 induced an immediate LAD2 cell degranulation, as shown by the rapid decrease of immunostaining of cytoplasmic avidin granules within 5 min of viral infection, and the induction of cellular degranulation progressed over the course of $2 \mathrm{~h}$ viral infection (Fig. 4a).

The observation of SARS-CoV-2-triggering a rapid MC degranulation (as short as $5 \mathrm{~min}$ ) indicates that viral binding might be 

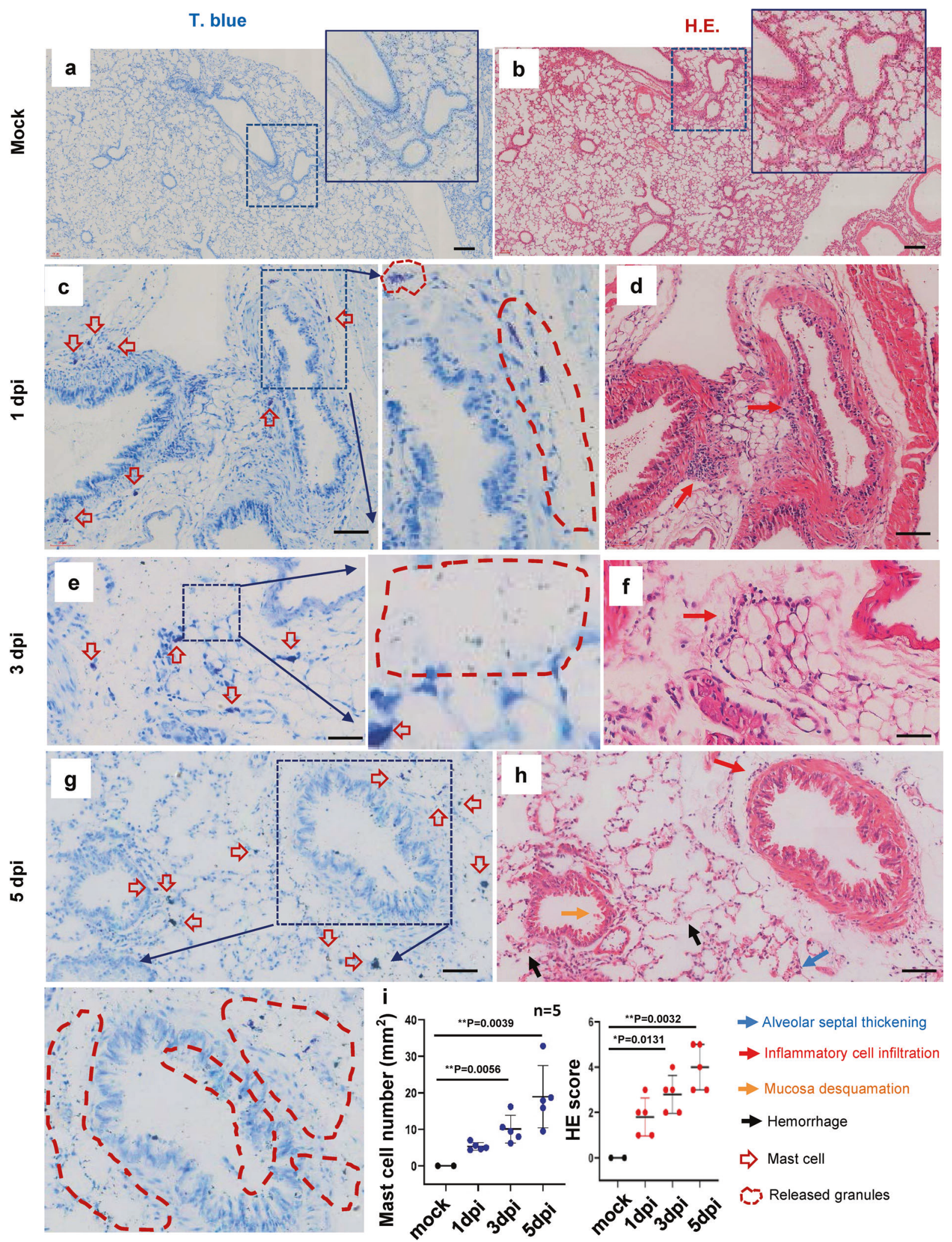
$\rightarrow$ Alveolar septal thickening
$\rightarrow$ Inflammatory cell infiltration
$\rightarrow$ Mucosa desquamation
$\rightarrow$ Hemorrhage
$\Rightarrow$ Mast cell
Released granules

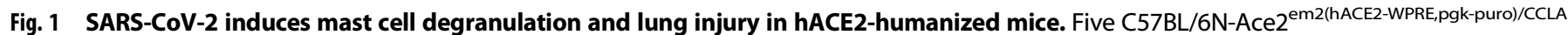
mice were intranasally infected with SARS-CoV-2 (strain 107) at a dose of $2 \times 10^{6} \mathrm{TCID}_{50}$, two mice were used as the mock-infections. The mice were euthanized at the $1 \mathrm{dpi}, 3 \mathrm{dpi}$ and $5 \mathrm{dpi}$, and the lung tissues were harvested for histological analysis. Toluidine blue staining was used to observe MCs and their degranulation ( $\mathbf{a}, \mathbf{c}$, e and $\mathbf{g}$ ), and the lung injury was observed by H.E. staining (b, d, $\mathbf{f}$ and $\mathbf{h}$ ), scale bar: $100 \mu \mathrm{m}$. (i) The pathological score was assessed according to the degree of lung tissue lesions and MC count in lung sections was calculated. ${ }^{*} p<0.05$ and ${ }^{* *} p<0.01$ are considered significant differences 


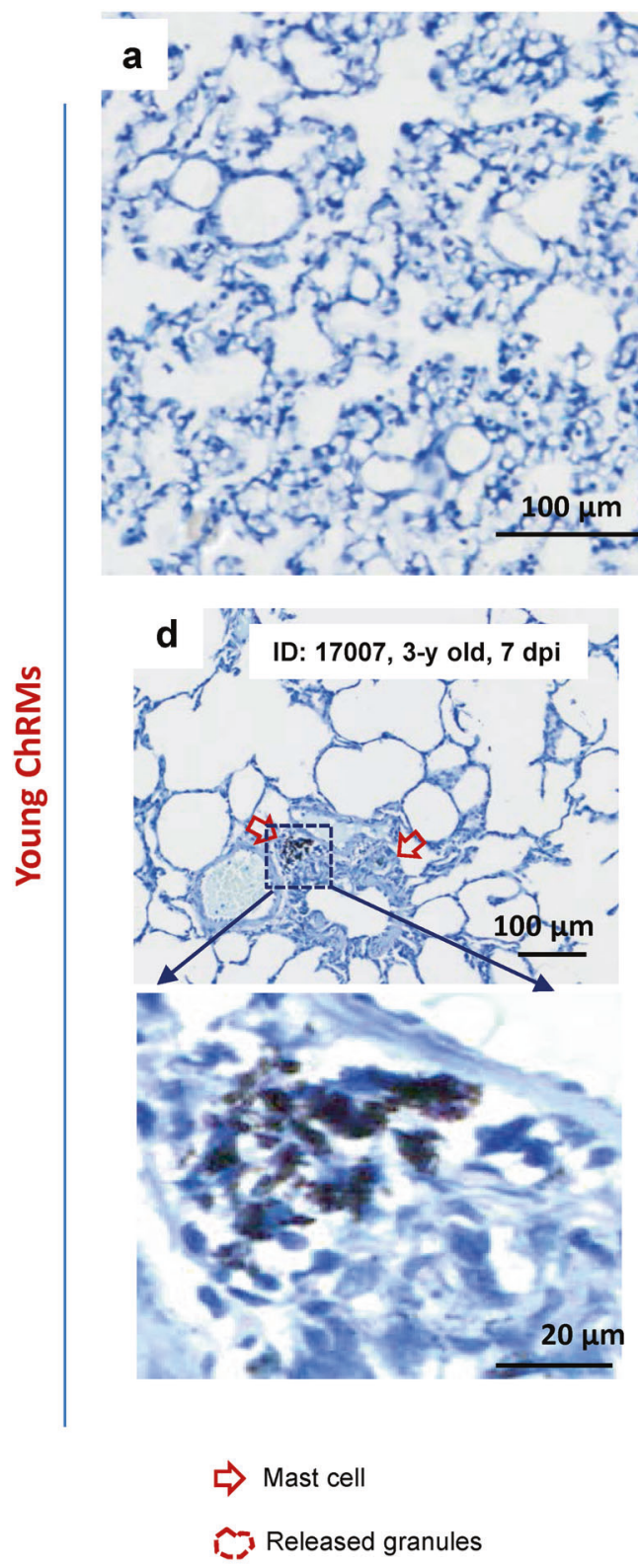

b ID: $15335,5-y$ old, 7 dpi
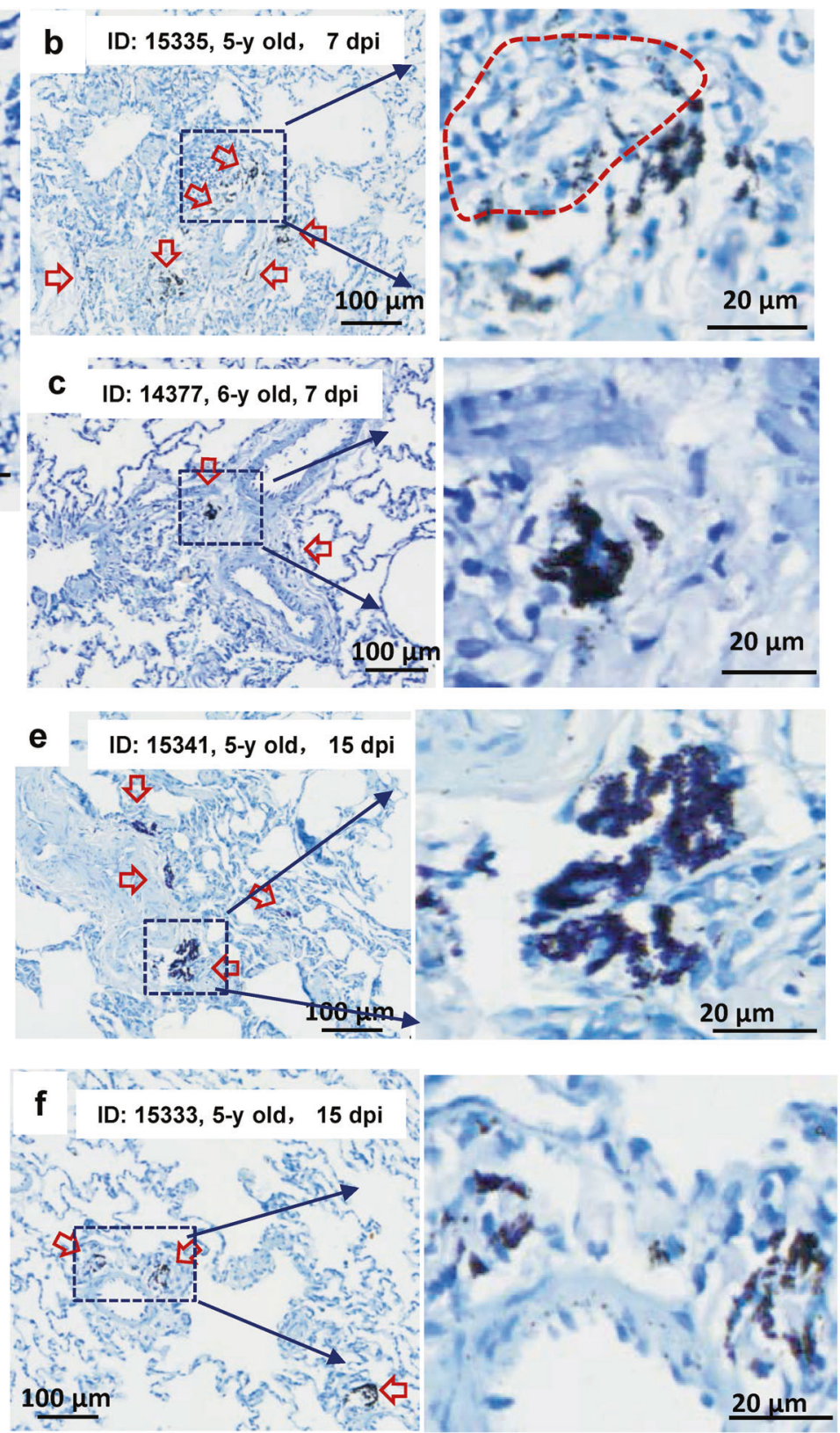

Fig. 2 SARS-CoV-2 induces mast cell degranulation in young chRMs. Young chRMs (3- to 6-year old) were anaesthetized by Zoletil 50 and intratracheally inoculated with SARS-CoV-2 $\left(1 \times 10^{7}\right.$ TCID $\left._{50}\right)$ in a $2 \mathrm{~mL}$ volume by bronchoscope. a Mock infection. The animals were euthanized at $7 \mathrm{dpi}(\mathbf{b}, \mathbf{c}, \mathbf{d})$ or $15 \mathrm{dpi}(\mathbf{e}, \mathbf{f})$ and the lung lobes were collected for histology analysis. Toluidine blue staining was used to observe MCs and their degranulation. Red arrow indicates the MCs

responsible for this event. LAD2 cells expressed ACE2 receptor as demonstrated by Western blotting (Fig. 4b) and flow cytometry (Fig. 4c), and were susceptible to SARS-CoV-2 and Spikepseudotyped lentivirus (NL4-3/S) (Supplementary Fig. 3). The recombinant receptor-binding domain (RBD) of SARS-CoV-2 Spike glycoprotein could bind to LAD2 cells at $4{ }^{\circ} \mathrm{C}$, and the priorblocking with anti-ACE2 specific antibody prevented Spike-RBD binding (Fig. 4d). Notably, Spike-RBD binding to ACE2 was accompanied by an immediate degranulation, as shown by the significant reduction of intracellular avidin intensity within 5 min of Spike-RBD treatment (Fig. 4e, Supplementary Fig. 4a), and the degranulation was progressively increased over the course of $2 \mathrm{~h}$ period of incubation (Fig. 4e). Spike-RBD-induced LAD2 degranulation was also quantified by the release of intracellular Tryptase and Chymase. The treatments of LAD2 cells with either Spike-RBD or Spike-pseudotyped lentivirus (Pseudo. Virus) for $2 \mathrm{~h}$ significantly increased the release of Tryptase and Chymase, and the compound $48 / 80$ (C48/80) was used as a positive experimental control for stimulation of MC degranulation (Fig. 4f).

To confirm that the specific interaction between Spike-RBD and ACE2 is key to trigger MC degranulation, we blocked the interaction by prior-treatment with anti-ACE2 antibody (Fig. 4d), and found that Spike-RBD treatment of LAD2 cells was no longer able to induce cell degranulation (Fig. 4g). The nucleocapsid protein treatment did not induce LAD2 degranulation (Supplementary Fig. 4b). A short $2 \mathrm{~h}$ stimulation with Spike-RBD stimulation did not show the obvious elevation of the de novo synthesis of cytokines in LAD2 cells, whereas the stimulation 


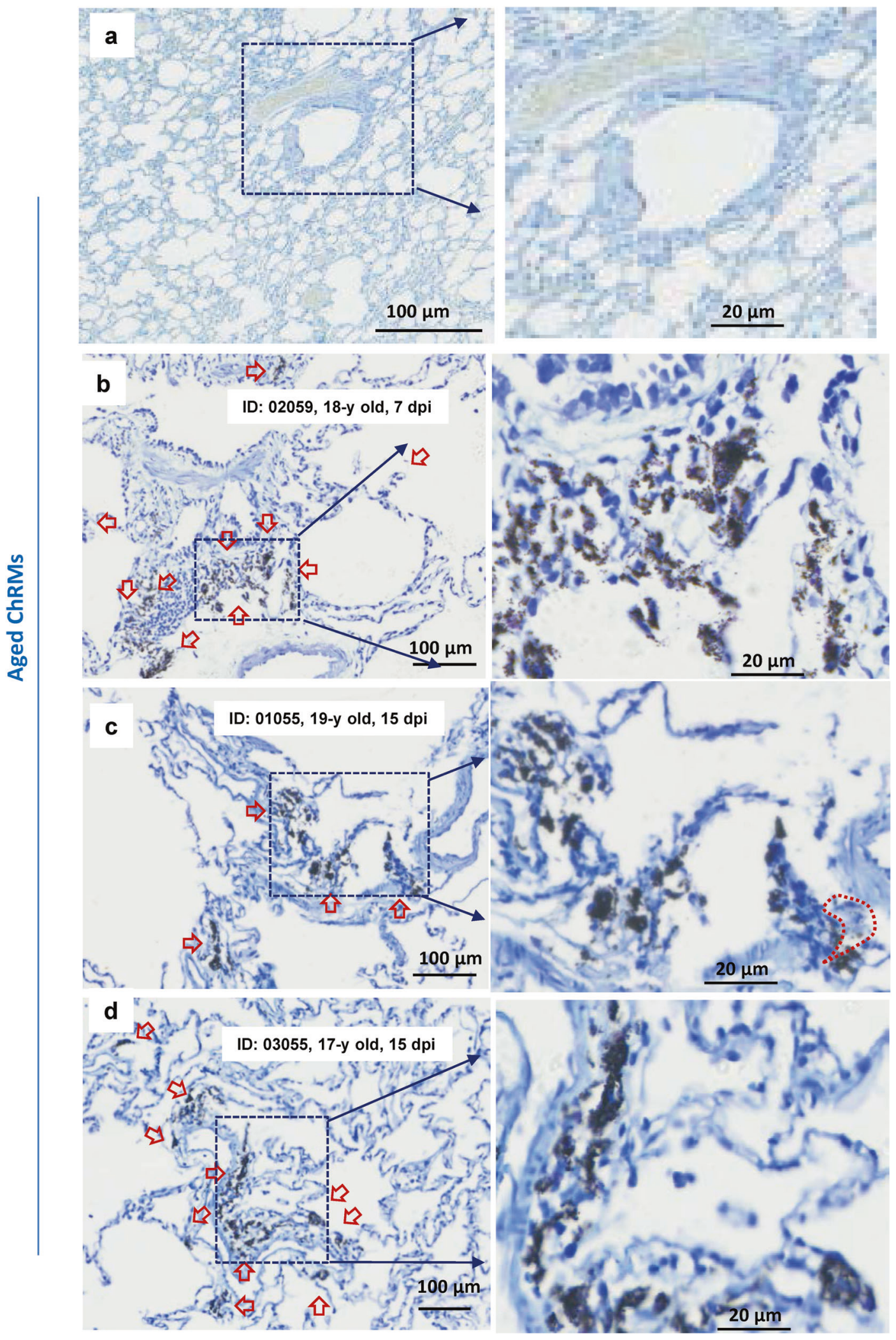

Fig. 3 SARS-CoV-2 induces mast cell degranulation in aged chRMs. Aged chRMs (17- to 19-year old) were anaesthetized by Zoletil 50 and intratracheally inoculated with SARS-CoV-2 $\left(1 \times 10^{7} \mathrm{TCID}_{50}\right)$ in a $2 \mathrm{~mL}$ volume by bronchoscope. a Mock infection. The animals were euthanized at $7 \mathrm{dpi}(\mathbf{b})$ or $15 \mathrm{dpi}(\mathbf{c}, \mathbf{d})$ and the lung lobes were collected for histology analysis. Toluidine blue staining was used to observe MCs and their degranulation. Red arrow indicates the MCs 
a

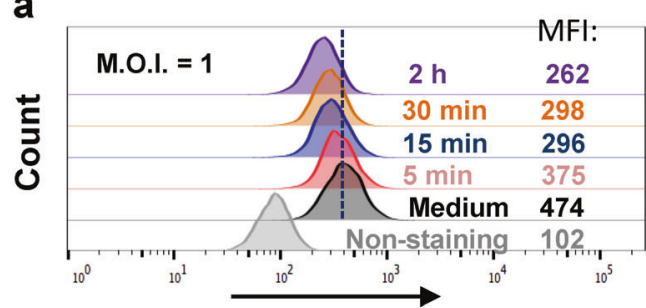

b

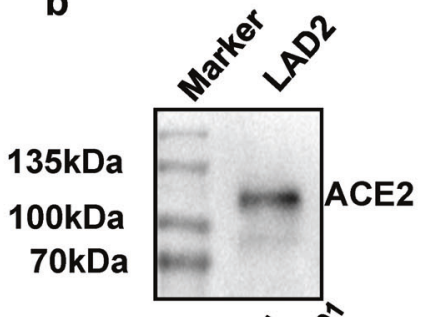

C

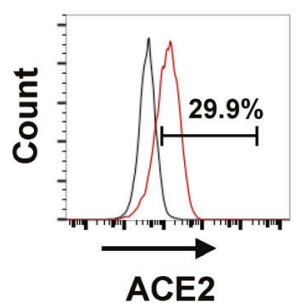

FITC-Avidin (log fluorescence)

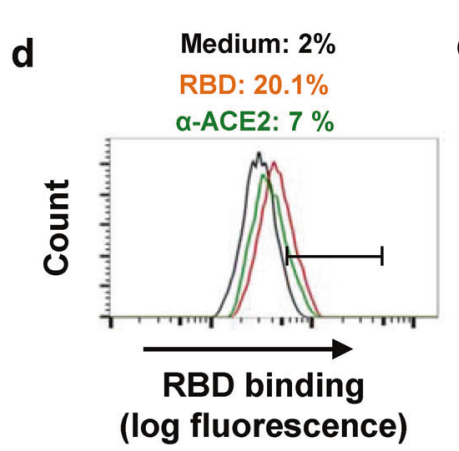

Tryptase

f

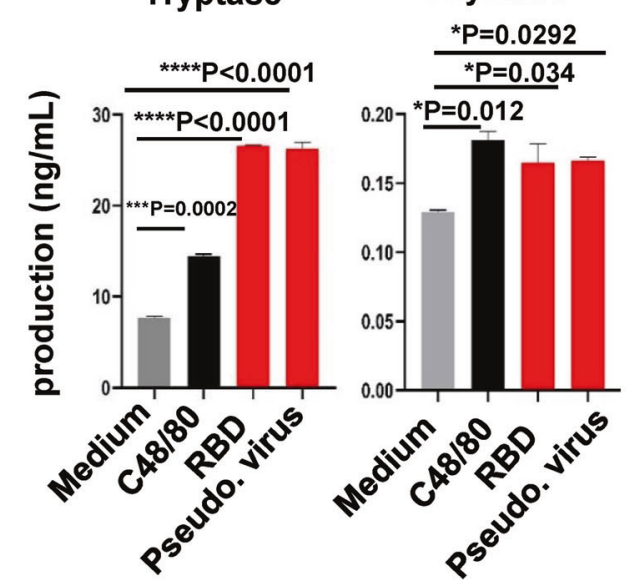

e

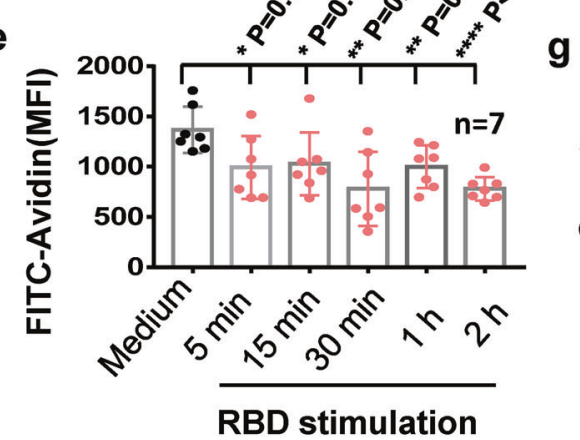

(log fluorescence)

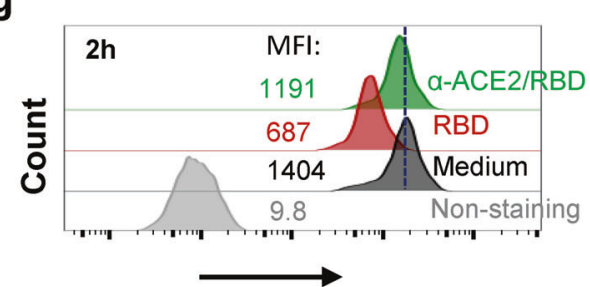

FITC-Avidin (log fluorescence)

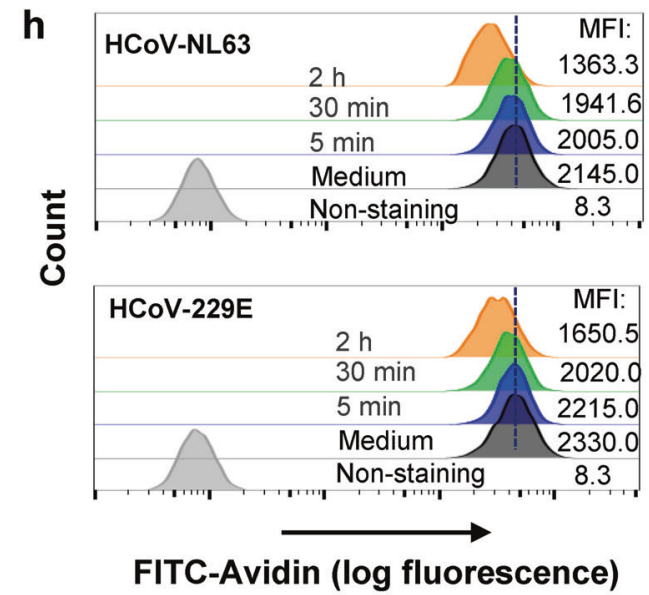

Fig. 4 The binding of Spike-RBD to ACE2 triggers a rapid MC degranulation. a SARS-CoV-2 (M.O.I.=1)-induced LAD2 degranulation, detected by flow cytometry with immunostaining the intracellular avidin. b, c ACE2 expression in LAD2 cells, detected with Western blotting and flow cytometry with immunostaining with specific antibodies. d Prior-blocking with anti-ACE2 antibody reduces RBD binding. LAD2 cells were incubated with anti-ACE2 antibody $(5 \mu \mathrm{g} / \mathrm{ml})$ at $37^{\circ} \mathrm{C}$ for $1 \mathrm{~h}$, then Spike-RBD $(5 \mu \mathrm{g} / \mathrm{ml})$ were added for binding at $4{ }^{\circ} \mathrm{C}$ for $1 \mathrm{~h}$, and the binding of Spike-RBD to LAD2 cells was detected with flow cytometry. e, $\mathbf{f}, \mathbf{g}$ Spike-RBD induces LAD2 degranulation. LAD2 cells were incubated with Spike-RBD $(5 \mu \mathrm{g} / \mathrm{ml})$ at $37^{\circ} \mathrm{C}$ for the indicated time, then cells were fixed with $4 \%$ paraformaldehyde and permeabilized and immunostained with anti-avidin-FITC at $4{ }^{\circ} \mathrm{C}$ for $1 \mathrm{~h}$, and analyzed with flow cytometry $(\mathbf{e}, \mathbf{g})$, results from 7 independent repeats were summarized and presented (e). The degranulated components Tryptase and Chymase were detected by ELISA (f). LAD2 cells were priortreated with anti-ACE2 antibody $(5 \mu \mathrm{g} / \mathrm{ml})$ at $37^{\circ} \mathrm{C}$ for $1 \mathrm{~h}$ before the Spike-RBD stimulation (g). h HCoV-NL63 and HCoV-229E induced LAD2 degranulation. LAD2 cells were incubated with HCoV-NL63 or HCoV-229E (M.O.I=1) for the indicated time, and cell degranulation was detected as above. One representative data from $3(\mathbf{a}, \mathbf{b}, \mathbf{f}, \mathbf{h})$ or $4(\mathbf{c}, \mathbf{d}, \mathbf{g})$ independent repeats are shown. Data are presented as mean \pm SD. ${ }^{*} p<0.05,{ }^{* *} p<0.01,{ }^{* * *} p<0.001$ and ${ }^{*} p<0.0001$ are considered significant differences. MFI mean fluorescence intensity

longer than $8 \mathrm{~h}$ induced productions of pro-inflammatory cytokines (Supplementary Fig. 5).

We also investigated whether other type of coronavirus could also induce MC degranulation. The coronaviruses of HCoV-NL63 and HCoV-229E were used to treat LAD2 cells $($ M.O.I = 1), the rapid MC degranluation was observed, as shown by the rapid decrease of immunostaining of cytoplasmic avidin granules within 5 min of viral stimulation, and the induction of cellular degranulation progressed over the course of $2 \mathrm{~h}$ viral infection (Fig. $4 \mathrm{~h}$ ).

Taken together, these data demonstrate that the binding of Spike-RBD of SARS-CoV-2 to ACE2 triggers an immediate MC degranulation, which is rapid and specific.
Transcriptome analysis reveals MC degranulation-induced remodeling of cellular signalings in human alveolar epithelial cells SARS-CoV-2 infection causes severe alveolar epithelial inflammation and barrier dysfunction. ${ }^{55,56}$ To test whether SARS-CoV-2triggered $\mathrm{MC}$ degranulation play a role, we performed transcriptome analysis in the Spike-RBD-induced MC degranulation model. The culture supernatant of Spike-RBD-treated LAD2 cells were harvested and used to treat A549 cells (an adenocarcinomic human alveolar basal epithelial cell line) for $24 \mathrm{~h}$, then the transcriptome in A549 cells were analyzed using standard protocols. Data from 3 independent repeats were summarized. The volcano plot displayed a total of 1667 up-regulated genes and 
a

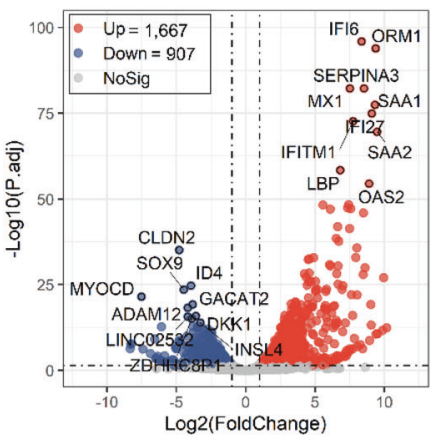

b

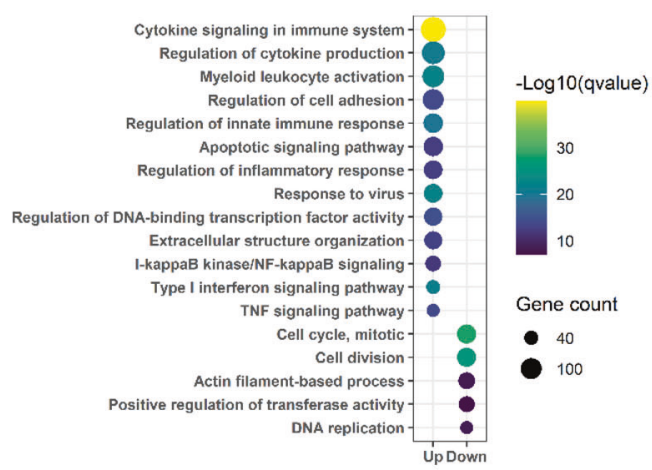

e
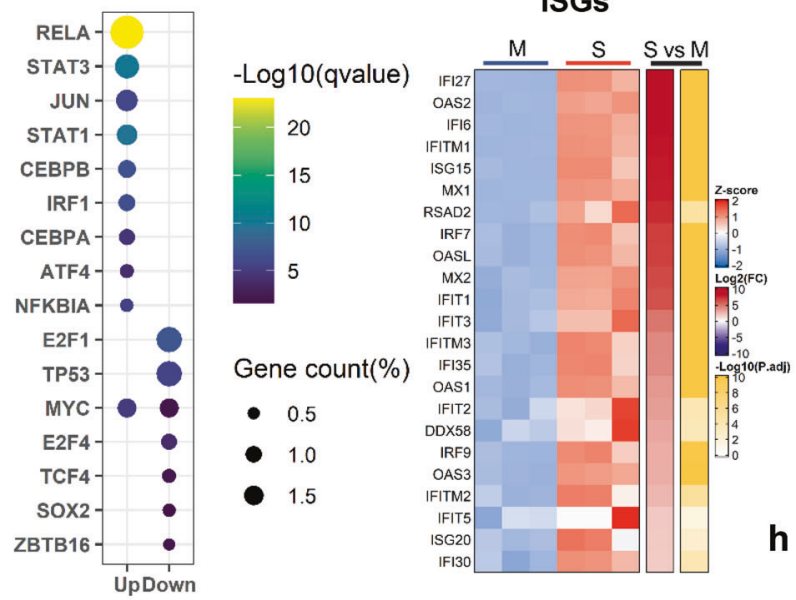

C

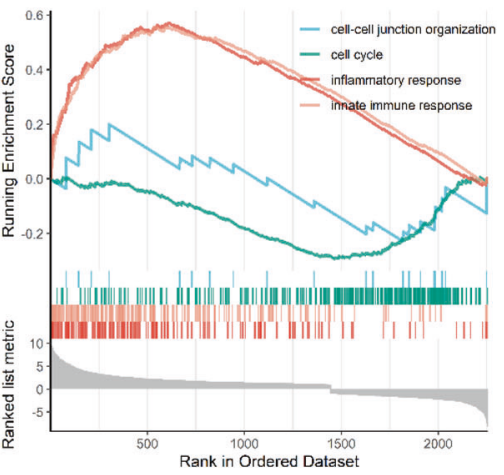

g

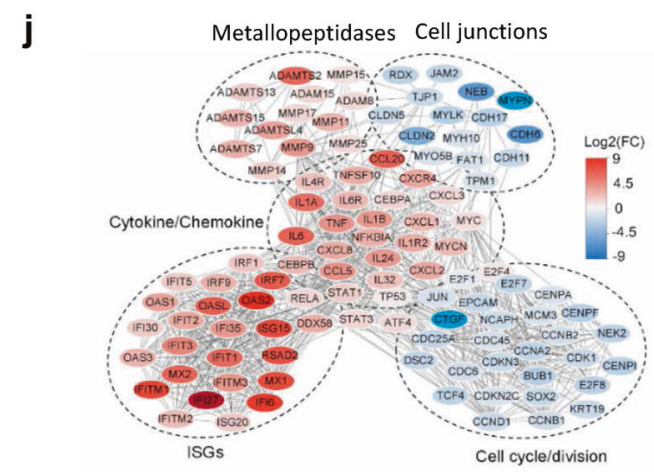

f Cytokine/Chemokine

h

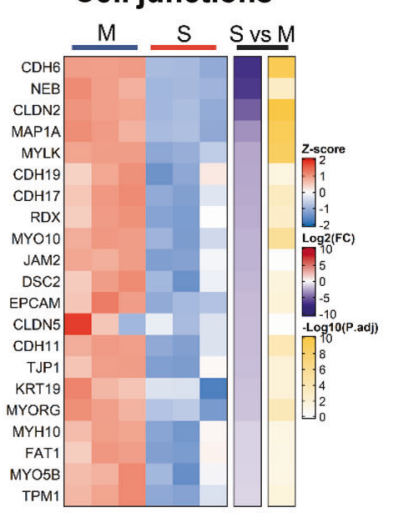

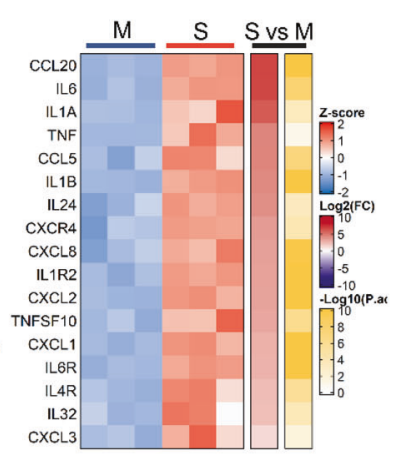

\section{i}

Cell cycle/division

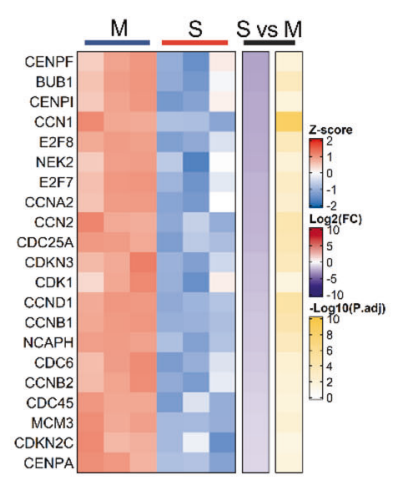

Fig. 5 Transcriptome analysis of A549 cells treated with LAD2/RBD supernatant. a Volcano plot of DEGs comparing LAD2/RBD supernatant versus medium. The symbols of top 10 up-regulated or down-regulated genes are shown. $\mathbf{b}$ GO functional enrichment analysis of DEGs. The color bar indicates the minus logarithm of $q$ values, and bubble size indicates the absolute gene counts enriched in a GO term. $c$ GSEA showing the distribution of gene sets that related to inflammatory response, innate immune response, cell-cell junction organization or cell cycle and the enrichment scores based on DEGs. d Transcription-factor enrichment analysis of DEGs. The color bar indicates the minus logarithm of $q$ values, and bubble size indicates the gene enrichment ratio regulated by a transcription factor. e-i Heatmaps showing relative expression level (left panel), fold change (middle panel), and adjusted $p$ values (right panel) for sets of ISGs (e), cytokine- and chemokinerelated genes (f), Metallopeptidase (g), cell junction-related genes (h), cell cycle- and division-related genes (i). M medium; S, LAD2/RBD supernatant. j A Protein-Protein interaction network analysis of the core DEGs. The color bar represents the fold change of protein-coded genes at transcriptome level

907 down-regulated genes after A549 cells were treated with supernatants from Spike-RBD-treated LAD2 cells (Fig. 5a). Gene ontology (GO) functional enrichment analysis of differently expressed genes (DEGs) showed obvious upregulation of gene sets that regulate cytokines signaling and production, the activation of myeloid and leukocyte, cell adhesion, innate immune and inflammatory response, and cell apoptotic signaling; in contrast, the gene sets that regulate cell cycle, cell division, and the actin filament-based cell movement processes were downregulated (Fig. 5b). The gene set enrichment analysis (GSEA) linked the up-regulated genes to the regulation of inflammatory and innate immune responses, and the down-regulated genes to the regulation of cell cycles and cell-cell junctions (Fig. 5c).

Transcription-factor enrichment analysis of DEGs showed that the up-regulated transcription factors were mainly those governing the immune and inflammatory response, e.g., RELA, 
STAT3, STAT1, JUN, CEBP- $\alpha / \beta$, IRF1 and ATF4; and the downregulated transcription factors were mainly linked to the regulations of cell proliferation and metabolisms, such as E2F1, E2F4, TP53 and ZBTB16 (Fig. 5d).

The core DEGs with highly differential expression were catalogized. The stimulation with LAD2/RBD supernatants induced elevated expression of antiviral immune genes in A549 cells, and a significant upregulation of genes for ISGs and IFN-I responses (e.g., IFI27, OAS2, IFI6, IFITM1, ISG15, MX1, RSAD2, IRF7, OASL, MX2, IFIT1 and IFIT3) (Fig. 5e) has been observed. Other prominent features were the upregulation of pro-inflammatory cytokines/chemokines, particularly CCL20, IL-6, IL 1 a, TNF, CCL5, IL $1 \beta$ and CXCL8 (IL-8) genes (Fig. 5f), and an upregulation of metallopeptidase-encoding genes (e.g., ADAMTS2 and MMPs) (Fig. 5g). LAD2/RBD supernatants induced a downregulation of a large number of genes encoding regulators of cell junctions and cell cycles/division. The downregulated core DEGs linked to cell junctions included those encoding for the tight junction proteins (e.g., CLDN2, CLDN5, TJP1 and JAM2), the cadherins (e.g., CDH6, CDH19, CDH17, CDH11, FAT1, $D S C 2$ and EP(AM), the cytoskeleton/microtubule-associated proteins (e.g., NEB, KRT19, RDX, TPM1 and MAP1A), and myosins (e.g., MYH10, MYO10, MYO5B, MYLK and MYORG) (Fig. 5h). The down-regulated core DEGs linked to cell cycles/division were mainly those encoding for the mitotic Serine/Threonine kinases and their regulators (e.g., BUB1, NEK2, CDK1; CDKN3, CDKN2C), centromere proteins (e.g., CENPF, CENPI, CENPA), regulation of transition phase of the cell cycle related transcription factors and cyclin family members (e.g., E2F7, E2F8; CCNA2, CCND1, CCNB1, $C C N B 2)$, genome replication regulators (e.g., $C D C 45, C D C 6, M C M 3)$, and cell division regulators (e.g., CCN2, CDC25A), etc (Fig. 5i). A Protein-Protein interaction network (PPI) analysis of the core DEGs was constructed to visualize the relationship between DEGs and between signaling pathways (Fig. $5 \mathrm{j}$ ).

Taken together, the transcriptome data reveal that SARS-CoV2-triggered $M C$ degranulation significantly alters multiple cellular signalings in human alveolar epithelial cells, particularly, MC degranulation upregulates immune responses and inflammation, and downregulates cellular signalings related to cell junction and cell division.

Inhibition of SARS-CoV-2-triggered MC degranulation abolishes the subsequent alveolar epithelial inflammation

To seek drug candidates that may modulate $M C$ degranulation and consequential cytokine release, we examined the known MC stabilizer Sodium cromoglicate (Sod. crom.), and the histamine receptor 1 (HR1) antagonists Ketotifen Fumarate (Ket.), Ebastine (Eba.) and Loratadine (Lor.), which are routinely used for treating human allergy. Besides of being antihistamines, Ket., Eba. (and its main metabolite carebastine) and Lor. (and its main metabolite desloratadine) can also be used as MC stabilizers to prevent degranulation and release of inflammatory mediators. ${ }^{57-63}$

Our results showed that the prior-treatments of LAD2 cells with Eba., Lor., Sod. crom. or Ket., blocked Spike-RBD- and/or Spikepseudotyped lentivirus- induced cell degranulation (Fig. 6a). Next, we thought to use these $\mathrm{MC}$ stabilizers to reduce the subsequent stimulation of inflammatory mediators from alveolar epithelial cells. The LAD2 cells were prior-treated with the representative MC stabilizers, the second generation of antihistamines Lor. and Eba., to block Spike-RBD-induced cell degranulation, then the cell culture supernatants were harvested to treat lung epithelial cells A549 (Fig. 6b). The direct stimulation of A549 cells with Spike-RBD did not induce obvious expression of pro-inflammatory cytokines, however the treatment of A549 cells with the LAD2/RBD supernatants induced production of extremely high level of IL-6, TNF-a, IL-8, IL-1 $\beta$, CCL20 and CCL5; when the Spike-RBD-induced LAD2 cell degranulation was blocked by Lor. or Eba., the harvested supernatant lost its capacity to induce pro-inflammatory cytokines (Fig. 6c; Supplementary Fig. 6).
To confirm the observed LAD2/RBD supernatant-induced production of pro-inflammatory cytokines is not an artifact in a single cell line, the human non-small cell lung cancer cells H1299 were used for stimulation with LAD2/RBD supernatants. The same results have been gotten, as LAD2/RBD supernatants signficantly induced the expression of IL-6, IL-8, IL-1 $\beta$, TNF- $\alpha$ in H1299 cells (Supplementary Fig. 7).

The disruption of integrity of alveolar epithelial barrier is associated with defects in the tight junction proteins. In keeping with the above transcriptome analysis data (Fig. 5h), we found that the stimulation of A549 cells with LAD2/RBD supernatants reduced the tight junction protein ZO-1, Jam-2, Claudin-5 and Occludin, but the direct stimulation with RBD proteins showed no effect; with prior-treatment of LAD2 with Lor. or Eba. to block degranulation, the harvested LAD2/RBD culture supernatants were unable to impair tight junction proteins (Fig. $6 \mathrm{~d}$ ). These suggest that specific factor or factors released into the LAD2/RBD supernatants acted on the tight junction proteins.

One of the possible candidates is Matrix Metallopeptidase 9 (MMP-9) which has significantly elevated expression and activity in COVID-19 patients. ${ }^{64,65}$ MMP-9 can impair the alveolar epithelial-endothelial capillary barrier by degrading the extracellular matrix, stimulate neutrophil and leukocyte migration, and promote inflammation. ${ }^{66}$ Keeping consistent with the above transcriptome analysis data (Fig. $5 \mathrm{~g}$ ), the LAD2/RBD supernatanttreated A549 cells had higher MMP9 expression levels, and after blocking LAD2 degranulation with Lor. or Eba., the LAD2/RBD supernatants lost its ability to induce MMP9 gene expression (Fig. 6e, f). MMP19 is another significantly up-regulated gene in our above RNA-Seq data (Fig. 5g). MMP19 is expressed in monocytes, macrophages, fibroblasts, and endothelial cells. ${ }^{67} \mathrm{We}$ also confirmed that LAD2/RBD supernatants-treated A549 cells had higher MMP19 expression and the blocking of LAD2 degranulation with Lor. or Eba. lost the induction of MMP19 gene expression (Fig. 6g). Taken together, these data demonstrate that the Spike-RBD-triggered MC degranulation can further induce the production of pro-inflammatory cytokines and MMPs from A549 alveolar epithelial cells. In keeping with this mechanistic interpretation, drugs that preventing $M C$ degranulation can reduce alveolar epithelial inflammation and protect the tight junction proteins integrity.

MC stabilizers reduce SARS-CoV-2-induced lung inflammation and prevent lung injury in mice

To test whether the above observation derived from in vitro study could be applied to the actual infection in vivo, we went back to the SARS-CoV-2 infection model of hACE-2 humanized mice. The C57BL/6N-Ace2 ${ }^{\text {em2(hACE2-WPRE,pgk-puro)/CCLA }}$ were treated with Lor. $(10 \mathrm{mg} / \mathrm{kg})$ or Eba. $(5 \mathrm{mg} / \mathrm{kg})$ via i.p. 1 day prior to intranasal infection with SARS-CoV-2 (strain 107) at a dose of $5 \times 10^{6} \mathrm{TCID}_{50}$, and then Lor. or Eba. were continued to be administered daily for 5 days until the mice were euthanized at 5 dpi (Fig. 7a).

In untreated controls, SARS-CoV-2 challenge induced $M C$ accumulation in the peri-bronchus, and the robust MC degranulation was evidenced by abundant released granules in peribronchus and scatted in alveolar spaces (Fig. 7b). The H.E. staining of the adjacent lung sections showed lung lesions including the alveolar septal thickening, inflammatory cell infiltration, fibrinous exudate, hyaline film formation, mucosa desquamation, and hemorrhage (Fig. 7c). The administration of Lor. and Eba. blocked MC degranulation (Fig. 7d, f), and greatly reduced lung lesions (Figs. 7e, g, h, i). Additionally, the administration of these two MC stabilizers significantly dampened SARS-CoV-2-induced inflammation, as evidenced by the greatly reduced productions of IL-6, TNFa, CCL20, CCL5, IL-8, IL-1 $\beta$, IFN- $\gamma$ and CRP (Fig. 8a). Viral replication was also monitored. Total RNAs were prepared from the left lobe, the right lobus superior, the right lobus medius, the right lobus inferior and the pulmonary azygos lobe of each mouse, and viral 
a

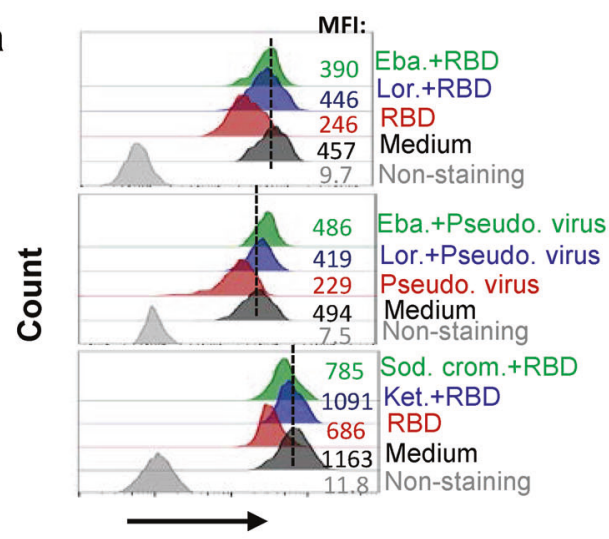

FITC-Avidin (log fluorescence)

b

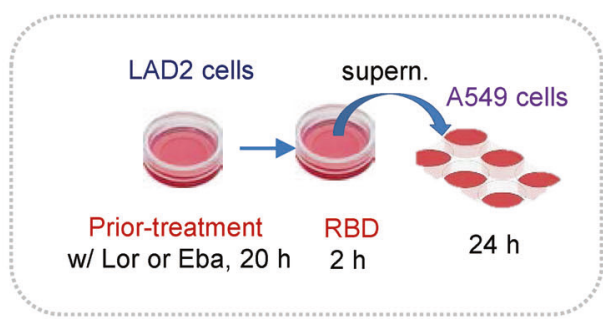

d

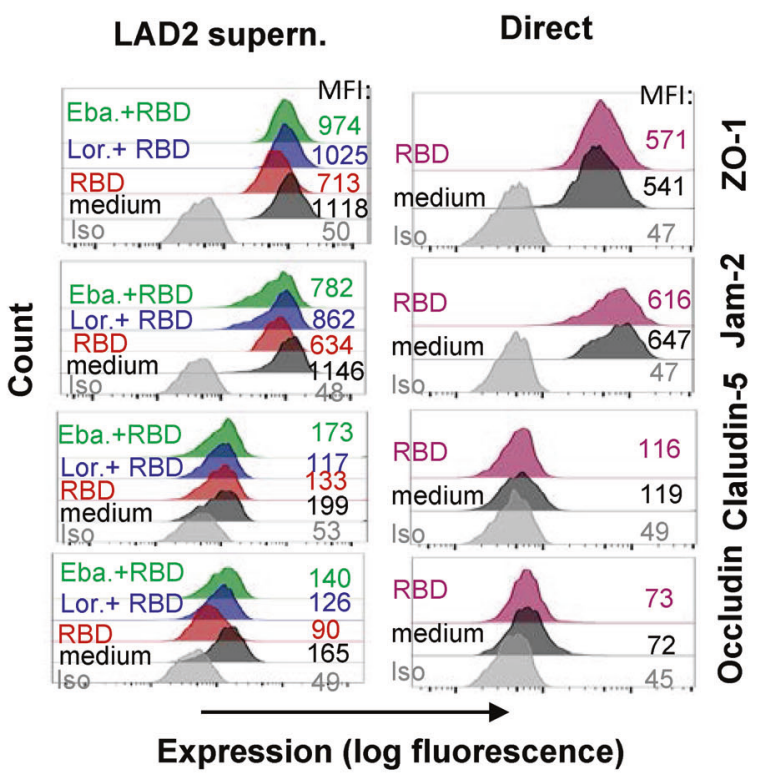

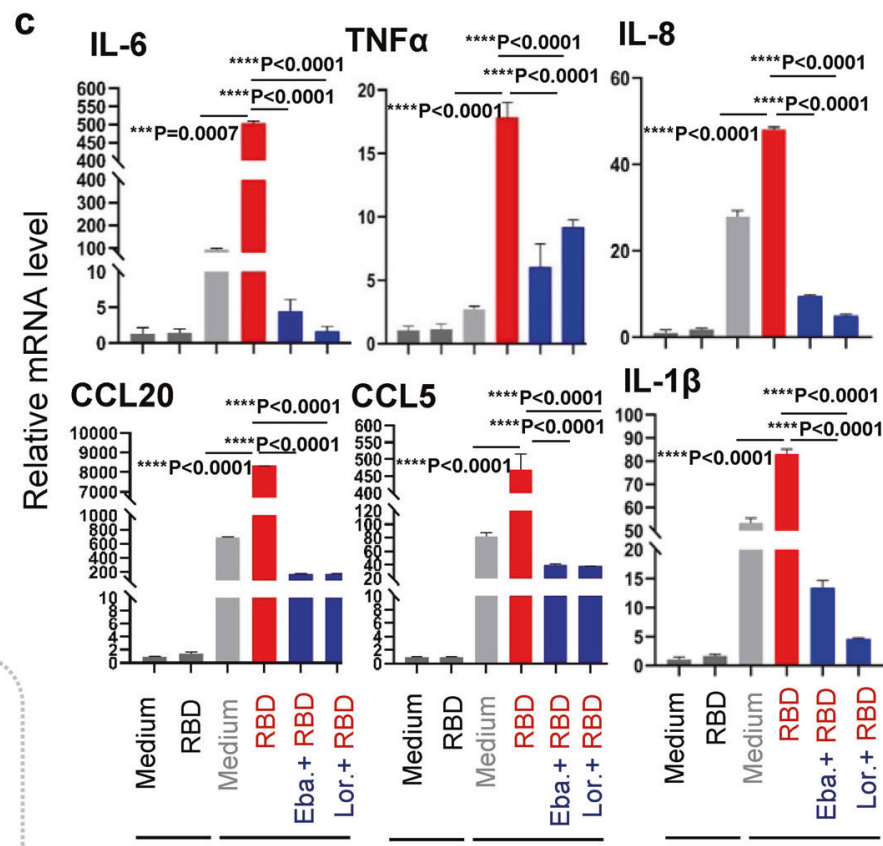

Direct LAD2 supern.Direct LAD2 supern. Direct LAD2 supern.

\section{Direct LAD2 supernatant from}

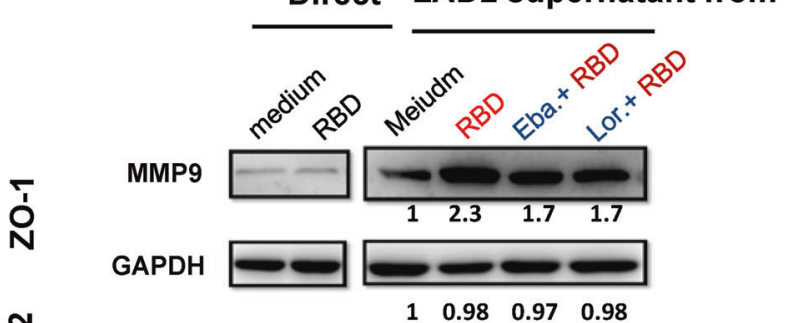

Direct LAD2 supern.

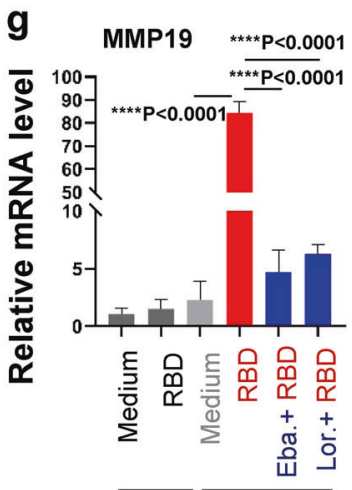

Direct LAD2 supern.

Fig. 6 Inhibition of MC degranulation abolishes alveolar epithelial inflammation. a Lor., Eba., Ket., or Sod. Crom. inhibits Spike-RBD or pseudotyped lentivirus-induced LAD2 cell degranulation. Cells were prior-treated with Lor. $(5 \mu \mathrm{g} / \mathrm{mL}), \mathrm{Eba}$. $(3 \mu \mathrm{g} / \mathrm{mL}), \mathrm{Ket}$. $(40 \mu \mathrm{g} / \mathrm{mL})$, or Sod. Crom. $(10 \mu \mathrm{g} / \mathrm{mL})$ for $20 \mathrm{~h}$, then were incubated with Spike-RBD $(5 \mu \mathrm{g} / \mathrm{ml})$ or Spike-pseudotyped lentivirus $\left(5 \mathrm{ng} \mathrm{p} 24^{\mathrm{Gag}}\right)$ at $37^{\circ} \mathrm{C}$ for the $2 \mathrm{~h}$, and cell degranulation was detected with Flow cytometry. $\mathbf{b}$ The illustration for treatments. LAD2 cells were prior-treated with or without Lor. $(5 \mu \mathrm{g} / \mathrm{mL})$ or Eba. $(3 \mu \mathrm{g} / \mathrm{mL})$ for $20 \mathrm{~h}$, then cells were treated with Spike-RBD $(5 \mu \mathrm{g} / \mathrm{ml})$ for $2 \mathrm{~h}$, and the culture supernatants were harvested to treat A549 cells for additional $24 \mathrm{~h}$, or A549 cells were directly treated with or without Spike-RBD for $24 \mathrm{~h}, \mathrm{c}$ the mRNA levels of IL-6, TNF- $\alpha$, IL-8, CCL20, CCL5 and IL-1 $\beta$ were quantified with real time q(RT-) PCR, and normalized to gapdh mRNA, d the expressions of ZO-1, Jam-2, Claudin-5 and Occludin were detected by immunstaining with specific antibodies and analyzed with flow cytometry, and (e, f), the expressions of MMP9 and MMP19 in A549 cells were measured with Western blotting or real time $q(R T-)$ PCR, and the gray intensity of gel was calculated by Image $J$ and normalized (e). One representative data from $3(\mathbf{c}, \mathbf{d}, \mathbf{e}, \mathbf{g})$ or $5(\mathbf{a}, \mathbf{f})$ independent repeats are shown. Data are presented as mean \pm SD. ${ }^{* * *} p<0.0001$ and ${ }^{* * * *} p<0.0001$ are considered significant difference (c, f). MFI mean fluorescence intensity (a, d) 
a

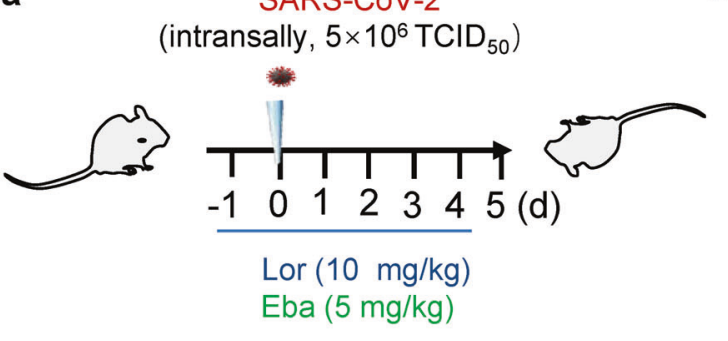

b T. Blue
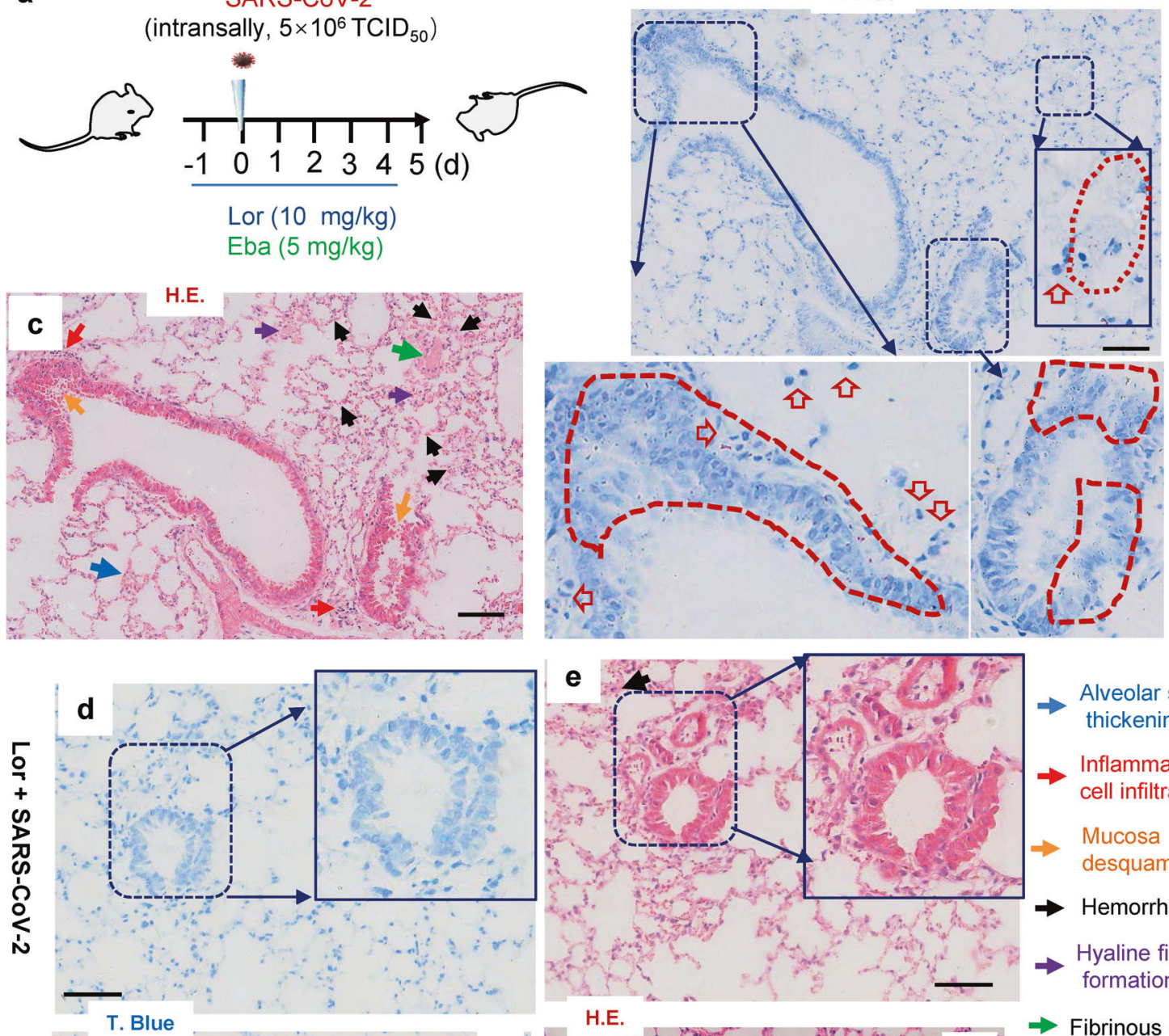

$\rightarrow$ Alveolar septal thickening

$\Rightarrow$ Inflammatory cell infiltration Mucosa desquamation

Hemorrhage

$\rightarrow$ Hyaline film formation
H.E.

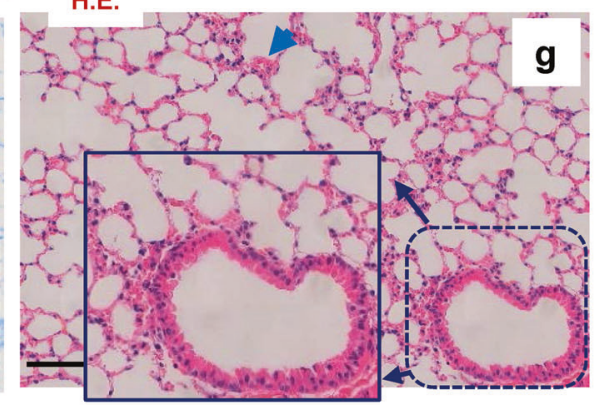

$\rightarrow$ Fibrinous exudate

$\Rightarrow$ Mast cell

시 Released granules

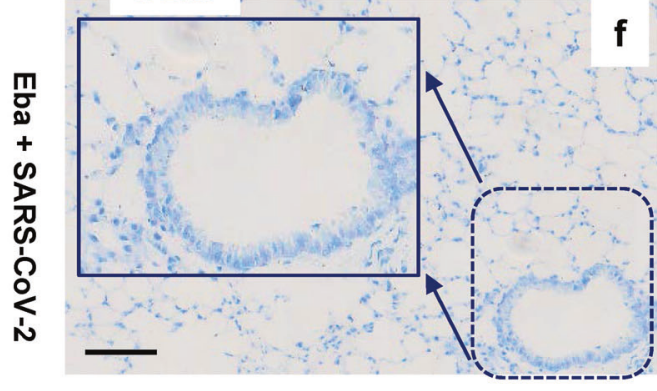

h

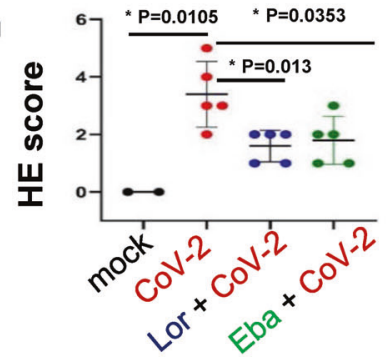

i

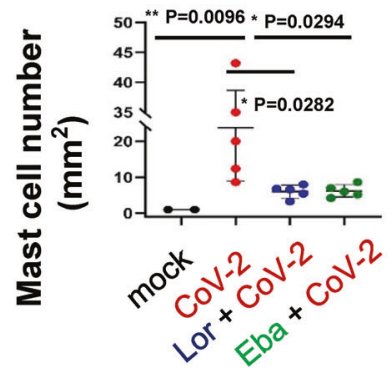

Fig. 7 MC stabilizers prevent lung injury in mice. a The illustration of mice treatment. C57BL/6N-Ace ${ }^{\text {em2(hACE2-WPRE,pgk-puro)/CCLA }}$ mice were prior- administered with or without Eba. $(5 \mathrm{mg} / \mathrm{kg})$ or Lor. $(10 \mathrm{mg} / \mathrm{kg})$ via i.p. 1 day before intranasal infection with SARS-CoV-2 (strain 107) at a dose of $2 \times 10^{6} \mathrm{TCID}_{50}$, and the Eba. and Lor. treatments were continued each day over the couse of infection. 5 mice for each treatment groups, and 2 mice without infection and drug treatment were used as the mock controls. Mice were euthanized and lung lobes were harvested for pathological analysis: Toluidine blue staining was used to observe MC degranulation, and the lung injury was observed via H.E. staining. Scale bar: $100 \mu \mathrm{m}$. h H.E. scores and (i) MC counts in lung section (5 dpi). ${ }^{*} p<0.05$ and ${ }^{* *} p<0.01$ are considered significant differences 

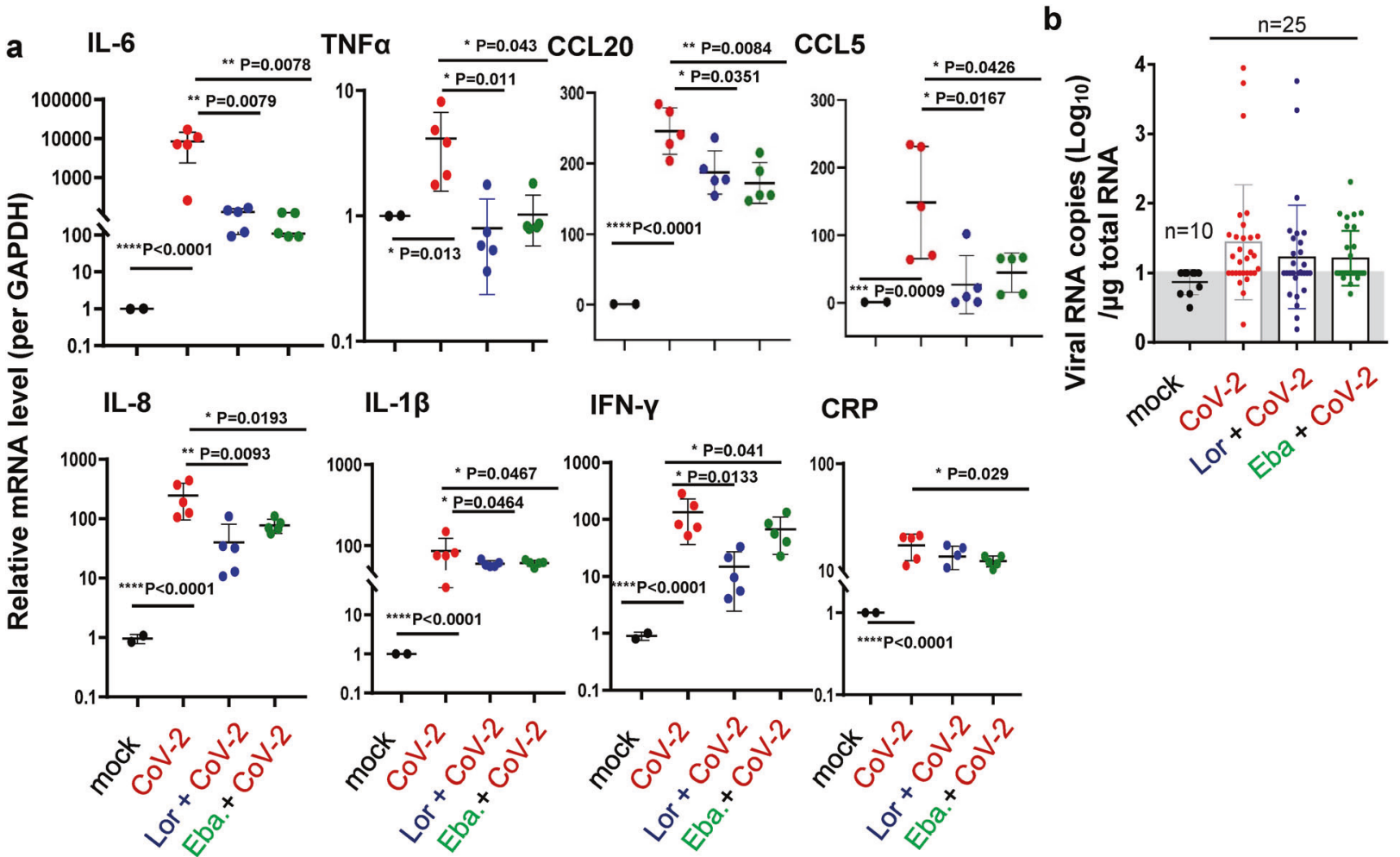

Fig. 8 MC stabilizers reduce SARS-CoV-2-induced inflammation. C57BL/6N-Ace2 ${ }^{\text {em2(hACE2-WPRE,pgk-puro)/CCLA }}$ mice were prior- administered with or without Eba. $\left(5 \mathrm{mg} / \mathrm{kg}\right.$ ) or Lor. $(10 \mathrm{mg} / \mathrm{kg})$ via i.p. 1 day before intranasal infection with SARS-CoV-2 (strain 107) at a dose of $2 \times 10^{6}$ $\mathrm{TCID}_{50}$, and the Eba. and Lor. treatments were continued each day over the couse of infection. 5 mice for each treatment groups, and 2 mice without infection and drug treatment were used as the mock controls. Mice were euthanized and lung lobes were harvested for analysis. a The mRNA levels of IL- 6 , TNF- $\alpha$, CCL20, CCL5, IL- 8, IL-1 $1 \beta$, IFN- $\gamma$ and CRP were quantified with q(RT-) PCR, and normalized to gapdh mRNA. b Viral replication was monitored by quantifying the expression of nucleocapsid gene. Data are presented as mean \pm SD. ${ }^{*} p<0.05,{ }^{* *} p<0.01$, ${ }^{* * *} p<0.001$ and ${ }^{* * * *} p<0.0001$ are considered significant differences

replication was measured by quantifying the expression of nucleocapsid gene. The mono-treatment with either Lor. or Eba. had not significantly reduced viral replication (Fig. 8b). Consistent with the idea that these drugs reduce SARS-CoV-2-induced lung inflammation and injury directly through preventing MC degranulation and subsequent inflammation, rather than indirectly by reducing virus load. Taken together, these data demonstrate that the administration of MC stabilizers reduces SARS-CoV-2-induced lung inflammation and prevents lung injury in mice.

\section{DISCUSSION}

In this study, we demonstrate a pivotal role of MCs in SARS-CoV-2induced epithelial inflammation and lung injury in vivo and elucidate its possible mechanisms in vitro. Besides of SARS-CoV-2, the other type of Coronavirus such as HCoV-NL63 and HCoV-229E could also induce MC degranlulation. It is possible that Coronavirusinduced MC degraulation to initiate epithelial hyper-inflammation represents a common mechanism for leading to organ/tissue damage. This point is worth further investigation.

The validation of MC stabilizers Eba. and Lor. can inhibit SARS-CoV2-induced hyper-inflammation and lung injury is highly significant, because it suggests an off-label use of MC stabilizers as immunomodulators to treat the severe cases of COVID-19. Our data in cell lines also demonstrate the blockage role of other MC stabilizers (e.g., Sodium cromoglicate and Ketotifen Fumarate) on Spike-RBD-induced $M C$ degranulation. The further clinical testing of their beneficial role of in the setting of SARS-CoV-2 infection would be much helpful in the treatment of lung injury in COVID-19 patients.

The strategical location at mucosa makes $M C$ being the sentinel to the exposed pathogens includuing SARS-CoV-2.
By releasing the soluble factors, $\mathrm{MCs}$ can recruit multiple types of immune cells to beneficially or detrimentally regulate immune inflammations. ${ }^{41,42}$ Given our findings of SARS-CoV-2 Spike-RBD induced rapid MC degranulation, one may speculate that the $M C$ activation and consequential inflammatory mediators release is one of the root-causes of lung inflammation and injury in COVID-19.

The phenotypes of MC degranulation-induced epithelial inflammation and lung injury in our study is concordant with the immunological and histopathological findings of COVID-19 patients and infected animals, ${ }^{44,68,69}$ indicating that SARS-CoV-2induced MC degranulation is a major cause that triggers hyperinflammation and lung injury. We find that $M C$ degranulation induces extremely high level of productions of pro-inflammatory cytokines and chemokines including IL-6, which has been shown to be an independent risk factor for disease severity and death of COVID-19. ${ }^{13,17,20-22}$

Spike-RBD-triggered $\mathrm{MC}$ degranulation provides an important route to disrupt alveolar barrier by inducing various types of metallopeptidases. LAD2/RBD supernatant promotes the expression of MMP9 in alveolar epithelial cells, which resembles the observation of significantly increased circulating MMP9 level in COVID-19 patients. ${ }^{64,65}$ In acute lung injury, the released MMP9 are able to promote degradation of the alveolar-capillary barrier, through probably action on tight junction proteins. ${ }^{66}$

The released components of SARS-CoV-2-induced MC degranulation contain Chymase and Typtase, which can also be used to explain virus-induced permanent damage to the alveoli epithelial cells and capillary endothelial cells. In inflammation or pathological conditions, the released Chymases from activated MCs are able to amplify local angiotensin-2 concentration to induce 
inflammatory leucocyte recruitment and endothelial dysfunction, which would further increase pulmonary vascular permeability and cause lung injury. ${ }^{70,71}$ Other types of viruses such as Dengue virus has been reported to trigger the release of Chymase and Typtase to breakdown the endothelial cell tight junctions to promote vascular permeability. ${ }^{72}$

SARS-CoV-2 infection can shutdown the expressions of cell cycle kinases (e.g., CDK1/2/5 and AURKA, etc) and result in cell cycle arrest between $S$ and G2 phases of the cell cycle. ${ }^{6,73}$ In our study, we find that Spike-RBD-triggered MC degranulation shutdown the expressions of various genes linking to the regulation of cell cycles/division. Besides, LAD2/RBD supernatants can directly suppress the expression of genes encoding the cytoskeleton/ microtubule-associated proteins and myosins, suggesting $M C$ degranulation induces the change of cytoskeleton organization. Our experimental data, again, consistent with the clinical observation that SARS-CoV-2 infection inducing changes of cytoskeletal-microtubule organization. ${ }^{68,73}$

It seems that the infection and replication of SARS-CoV-2 in MCs is not necessarily required for triggering degranulation, as the stimulation of Spike-RBD can trigger MC degranulation. MC cells express ACE2 receptor and are permissive for SARS-CoV-2 replication. Whether SARS-CoV-2 replication in MCs leads to the de novo synthesis of inflammatory mediators for the secondary release is worthy of further investigation. Additionally, SARS-CoV2-induced MC degranulation may also be used to explain the disease severity in aged individuals. We find that SARS-CoV-2 induces a more robust MC degranulation in aged chRMs, which could link the severer cytokine storm and higher immune cell infiltration in aged adults and RMs. ${ }^{52,69,74,75}$

The limitation of our study is that the mono-administration of Eba. or Lor. shows less effect in directly suppressing viral replication in lungs of ACE2-humanized mice. A combination of MC stabilizers with antiviral drugs such as the RNA polymerase inhibitors Remdesivir and Favipiravir, ${ }^{76-79}$ may provide a more optimal treatment strategy for both dampening inflammation and clearing viruses at the same time.

In summary, we demonstrate that SARS-CoV-2 triggers lung MC degranulation, which induces the remodeling of various cellular signalings in human alveolar epithelial cells, particularly, MC degranulation induces the alveolar epithelial inflammation and leads to the consequent disruption of tight junction proteins; importantly, we find that the clinically used MC degranulation stabilizers Eba. and Lor. are potent agents at reducing virusinduced production of pro-inflammatory factors and preventing lung injury. Our finding uncovers a potentially novel mechanism of SARS-CoV-2 infection initiates alveolar epithelial inflammation and induces lung Injury. Significantly, our results suggest a potential off-label use of MC stabilizers as immunomodulators to treat the severe cases of COVID-19.

\section{MATERIALS AND METHODS}

Ethics statement

All animal experiments were approved by the Institutional Animal Care and Use Committee of Guangzhou Institutes of Biomedicine and Health and Kunming Institutes of Zoology, Chinese Academy of Sciences, and the first Affiliated Hospital of Guangzhou Medical University. The SARS-CoV-2 animal model experiments and protocols were also discussed explicitly and extensively with biosafety officers and facility managers. All animal experiments and wild type virus were conducted within the animal biosafety level 3 (ABSL-3) facility.

\section{Cells and viruses}

Human mast cell LAD2 and Human non-small cell lung cancer cells H1299 were cultured in RPMI 1640 medium (Gibco) containing $10 \%$ fatal bovine serum (FBS) (Gibco) with $100 \mathrm{U} / \mathrm{mL}$ penicillin and
$100 \mu \mathrm{g} / \mathrm{mL}$ streptomycin. Human hepatoma cell line Huh-7 was cultured in DMEM medium (Gibco) containing 10\% FBS (Gibco) with $100 \mathrm{U} / \mathrm{mL}$ penicillin and $100 \mu \mathrm{g} / \mathrm{mL}$ streptomycin. For degranulation, LAD2 cells were grown in StemPro-34 medium (Gibco) supplemented with $100 \mu \mathrm{g} / \mathrm{ml}$ stem cell factor (Novoprotein), $100 \mu \mathrm{g} / \mathrm{ml} \mathrm{IL-6}$ (Novoprotein), nutrient supplement (NS) (Gibco), $100 \mathrm{U} / \mathrm{ml}$ penicillin (Invitrogen), $100 \mu \mathrm{g} / \mathrm{ml}$ of streptomycin (Invitrogen) and $2 \mathrm{mM}$ L-Glutamine (Gibco). Adenocarcinomic human alveolar basal epithelial cells (A549) was cultured in DMEM/F12 medium (Gibco) containing 10\% FBS (Gibco) with $100 \mathrm{U} / \mathrm{mL}$ penicillin and $100 \mu \mathrm{g} / \mathrm{mL}$ streptomycin.

The 107 strain of SARS-CoV-2 was provided by Guangdong Provincial Center for Disease Control and Prevention, Guangdong Province of China. The Coronavirus HCoV-NL63 (NR-470) and HCoV-229E (VR-740) were purchased from the American Type Culture Collection (ATCC). Pseudotyped virus was generated by EZ Trans cell transfection reagent (Life iLab, AC04L082) -mediated cotransfection of HEK293T cells with the Spike-expressing plasmid pcDNA3.1-2019-nCoV-S-IRES (strain 2019-nCoV WIV04) and pNL43. Luc. $\Delta \mathrm{R} \Delta \mathrm{E} .{ }^{80}$ These two plasmids are provided by Dr. Lu Lu (Fudan University, Shanghai, China). Harvested supernatants of transfected cells that contained viral particles were aliquoted and stored at $-80^{\circ} \mathrm{C}$.

ACE2-humanzied mice and rhesus macaques experiments 3-4 months old C57BL/6N-Ace2 ${ }^{\text {em2(hACE2-WPRE,pgk-puro)/CCLA }}$ mice were provided by Guangzhou Institutes of Biomedicine and Health, Chinese Academy of Science. ${ }^{47}$ The mice were randomly assigned to each group and the ratio of females to males was 1:1. Mice ( 5 for each groups) were infected nasal inhalation with SARSCoV-2 (strain 107) $\left(5 \times 10^{6} \mathrm{TCID}_{50}\right)$ for indicated times. The same amount of PBS was inoculated as the mock infection. In some mice, ebastine $(5 \mathrm{mg} / \mathrm{kg})$ or loratadine $(10 \mathrm{mg} / \mathrm{kg}$ ) (both from Sigma-Aldrich) was administered 1 day before infection and the treatments were continued each day over the course of infection. The lungs were collected on the day of euthanization for pathological, virological and immunological analysis.

For Ad5-hACE2-transduced BALB/c mice, ${ }^{51}$ specific pathogenfree 6-10 weeks old male and female (ratio $=1: 1$ ) BALB/c mice were lightly anaesthetized with isoflurane and transduced intranasally with $2.5 \times 10^{8}$ fluorescence focus units (FFU) of Ad5ACE2 in $75 \mu \mathrm{L}$ DMEM. Five days post-transduction, mice were infected intranasally with $7 \times 10^{4} \mathrm{TCID}_{50}$ SARS-CoV-2 in a total volume of $50 \mu \mathrm{L}$ DMEM. The same amount of DMEM was inoculated as the mock infection. The SARS-CoV-2 strains used in this experiment were isolated from COVID-19 patients in Guangzhou (Accession numbers: MT123290). At the indicated time, the lungs of mice were collected on the day of euthanization for histology analysis.

For monkey study, eight Chinese rhesus macaques (chRMs) (Macaca mulatta) (all male), including young group (3- to 6-year old) and aged group (17- to 19-year old), were anaesthetized by Zoletil 50 (Viabac, France) and intratracheally inoculated with SARS-CoV-2 (virus stain 107) $\left(1 \times 10^{7} \mathrm{TCID}_{50}\right)$ in a $2 \mathrm{~mL}$ volume by bronchoscope. The animals were euthanized at 7 or $15 \mathrm{dpi}$ and the lung lobes were collected for histology analysis. ${ }^{52}$

\section{Histology}

Animal's lungs were fixed in zinc formalin. For routine histology, tissue sections ( $\sim 4 \mathrm{~m}$ each) were stained with Hematoxylin and Eosin or Toluidine blue. The sections were analyzed with the Motic Digital Scanning apparatus (BA600Mot-4C-VM). The pathological score was assessed according to the degree of lung tissue lesions including alveolar septal thickening, hemorrhage, inflammatory cells infiltration, and consolidation. The semiquantitative assessment were performed as follows, ${ }^{47} 0$ : no alveolar septal thickening; 1: alveolar septal thickening was very mild, the area of alveolar septal thickening, hemorrhage and inflammatory cells 
infiltration was less than 10\%; 2: when alveolar septal thickening was mild, the area of alveolar septal thickening, hemorrhage and inflammatory cells infiltration was $10-25 \%$; 3 : when alveolar septal thickening was moderate, the area of alveolar septal thickening, hemorrhage, inflammatory cells infiltration, hyaline film formation, mucosa desquamation and fibrinous exudate was 25-50\%; 4: when alveolar septal thickening was marked, the area of alveolar septal thickening, hemorrhage, inflammatory cells infiltration, hyaline film formation, mucosa desquamation and fibrinous exudate was 50-75\%; 5: when alveolar septal thickening was very marked, the area of alveolar septal thickening, hemorrhage, inflammatory cells infiltration, hyaline film formation, mucosa desquamation and fibrinous exudate was greater than $75 \%$.

\section{Spike-RBD protein binds to LAD2 cells}

LAD2 cells $\left(3 \times 10^{5}\right)$ were incubated with Spike-RBD protein $(5 \mu \mathrm{g} / \mathrm{mL}$, Genscript, Z03483) in adherent buffer $(1 \mathrm{mM} \mathrm{CaCl}$, $2 \mathrm{mM} \mathrm{MgCl} 2$ and $5 \% \mathrm{BSA}, \mathrm{pH}$ 7.4) for $1 \mathrm{~h}$ at $4{ }^{\circ} \mathrm{C}$. The cells were then fixed with $4 \%$ paraformaldehyde (Sigma-Aldrich) for 30 min at room temperature and stained with anti-His-tag antibodies (Abmart, M30111S). Subsequently, the cells were stained with goat anti-mouse Alexla Fluor 488-conjugated secondary antibodies (Invitrogen, A11001), and were detected with flow cytometry (BD Accuri (6) and analyzed with the FlowJo 7.6.1 software. In some experiments, LAD2 cells were prior-blocked with anti-ACE2 antibody $\left(5 \mu \mathrm{g} / \mathrm{mL}\right.$, R\&D Systems, AF933) for $1 \mathrm{~h}$ at $37^{\circ} \mathrm{C}$ before the incubation with Spike-RBD protein.

LAD2 cell degranulation

LAD2 cells $\left(3 \times 10^{5}\right)$ were exposed to Spike-RBD protein (Genscript) $(5 \mu \mathrm{g} / \mathrm{mL})$, nucleocapsid protein $(5 \mu \mathrm{g} / \mathrm{mL})$, SARS-CoV-2 (M.O.I. = 1) (strain 2019-nCoV WIV04), or HCoV-NL63 (ATCC, NR-470) and HCoV-229E (ATCC, VR-740) (M.O.I.=1) for the indicated times. Mast cell degranulation activator compound 48/80 (C48/80) $(4 \mu \mathrm{g} / \mathrm{ml})$ (Sigma, C2313) was used as the control. Cells were fixed with $4 \%$ paraformaldehyde (Sigma-Aldrich) at room temperature for $30 \mathrm{~min}$, and washed 3 times with PBS, then cells were incubated with anti-avidin-FITC $(500 \mathrm{ng} / \mathrm{mL}$, Invitrogen, A821) which was diluted in permeabilized buffer (1\% FBS and $0.2 \%$ Triton X-100 in PBS) at $4{ }^{\circ} \mathrm{C}$ for $1 \mathrm{~h}$. After washing, cells were detected with BD Accuri $\mathrm{C} 6$ and analyzed with FlowJo. In some experiments, Loratadine $(5 \mu \mathrm{g} / \mathrm{mL}$, Selleck), Ebastine $(3 \mu \mathrm{g} / \mathrm{mL}$, Selleck), Ketotifen Fumarate $(40 \mu \mathrm{g} / \mathrm{mL}$, Yuanye Biology, China, S46226), or Sodium cromoglycate $(10 \mu \mathrm{g} / \mathrm{mL}$, Sigma, 15826-37-6) was used to prior-treat cells for $20 \mathrm{~h}$ before stimulation with SpikeRBD protein. The LAD2 cell culture supernatants were harvested for quantifying the released components of Chymase and Tryptase with ELISA kits according the manufacturer's instructions instruments (Lunchangshuo Biotech, Tryptase: SU-B10563; Chymase: SU-B16617).

Inflammatory cytokine assay

A549 cells $\left(3 \times 10^{5}\right)$ were treated with LAD2 culture supernatant $(250 \mu \mathrm{L})$ for $24 \mathrm{~h}$, then cells were harvested. The cytokines were determined either by quantifying the production of mRNAs or intracellular immuno-staining with specific antibodies. For immunostaining assay, A549 cells were added leukocyte activation cocktail containing BD GolgiPlug (BD, 550583) and cultured for $6 \mathrm{~h}$. Following activation, cells were washed with FACS buffer. BD Cytofix/Cytoperm solution (BD, 554722) was used for the simultaneous fixation and permeabilization of cells for $20 \mathrm{~min}$ at $4{ }^{\circ} \mathrm{C}$ before intracellular cytokine staining. Antibodies diluted in Perm/ Wash buffer was added and cells were further incubated at $4{ }^{\circ} \mathrm{C}$ for overnight. After washing, cells were resuspended in FACS buffer to flow cytometric analysis (BD LSRFortessa). Cytokine antibodies against the flowing markers were used: Alexa Fluor 647-IL-1 $\beta$ (Biolegend, JK1B-1), PE-IL-6 (BD, MQ2-6A3), BV421-IL-8 (BD, G265-8).
Real time (RT-) PCR

Total RNAs from cells were extracted by using TRlzol Reagent (Invitrogen) and then reverse transcribed into CDNA with synthesis Kit (TOYOBO, FSQ-301), according to the manufacturer's instructions. Real-time PCR was carried out by using the SYBR qPCR Mix (Genestar, A33-101) with the following thermal cycling conditions: initial denaturation at $95^{\circ} \mathrm{C}$ for $2 \mathrm{~min}$, amplification with 40 cycles of denaturation at $95^{\circ} \mathrm{C}$ for $15 \mathrm{~s}$, primer annealing at $60^{\circ} \mathrm{C}$ for $15 \mathrm{~s}$, and extension at $72^{\circ} \mathrm{C}$ for $30 \mathrm{~s}$. The data were analyzed by SYBR green-based semi-quantification and normalized with GAPDH. Real-time PCR was performed on the Bio-Rad CFX96 Real-Time PCR system. The mice extracted RNAs were used to measure the copies of nucleocapsid gene SARS-CoV-2 using THUNDERBIRD Probe One-step qRT-PCR Kit (Toyobo). The standard samples were purchased from the National Institute of Metrology of China. The primers and probes for (RT-) PCR were listed in Supplementary Table 1.

\section{Western blotting}

LAD2 or A549 cells were lysed for $1 \mathrm{~h}$ at $4{ }^{\circ} \mathrm{C}$ in lysis buffer (Beyotime). After centrifugation for $10 \mathrm{~min}$ at $12,000 \mathrm{~g}$, the supernatant was boiled in reducing SDS sample loading buffer and analyzed by SDS-PAGE. The anti-ACE2 antibody (Abcam, EPR4435), anti-MMP9 antibody (signal antibody, JA80-73), antiGAPDH antibody (Abcam, ab82633), and the horseradish peroxidase-conjugated secondary antibody were used in Western blotting.

\section{Flow cytometry}

The expression of ACE2 in LAD2 was determined by immunostaining with PE-labeled rabbit anti-ACE2 (Bioss, bs-1004R) and detecting with flow cytometry (BD Accuri C6). For detecting tight junction proteins ZO-1, Occludin, Claudin-5 and JAM2 in A549 cells, cells were blocked with 5\% BSA in PBS for $1 \mathrm{~h}$ at room temperature then incubated with primary antibodies for $2 \mathrm{~h}$ at $4{ }^{\circ} \mathrm{C}$. Primary antibodies against ZO-1 (Invitrogen, 402200), Occludin (Invitrogen, OC-3F10), Claudin-5 (Invitrogen, 4C3C2) and JAM-2 (Abcam, EPR2489), were used. A permeabilizing agent (1\% FBS and $0.2 \%$ Triton X-100 in PBS) was used for ZO-1 intracellular staining. Cells were washed with FACS buffer and then incubated with Alexa Flour 488-labeled goat anti-rabbit or goat anti-mouse IgG (Invitrogen, A11034; Invitrogen, A11001) for $1 \mathrm{~h}$ at $4{ }^{\circ} \mathrm{C}$, then cells were analyzed with flow cytometry (BD Accuri (6).

RNA sequencing and data analysis

A549 cells were treated with LAD2 cell culture supernatants for $24 \mathrm{~h}$. Total RNAs were extracted using Trizol (Invitrogen) according to the manufacturer's protocol, and ribosomal RNA removed using QIAseq FastSelect-rRNA HMR Kits (QIAGEN, Germany). Fragmented RNAs (average length $\sim 200 \mathrm{bp}$ ) were subjected to first strand and second strand CDNA synthesis, followed by adaptor ligation and enrichment with a low-cycle according to the instructions of NEBNext UltraTM RNA Library Prep Kit for Illumina (NEB, USA). The purified library products were evaluated using the Agilent 2200 TapeStation and Qubit2.0 (Life Technologies, USA). The libraries were paired-end sequenced (PE150, Sequencing reads were $150 \mathrm{bp}$ ) at Guangzhou RiboBio Co., Ltd. (Guangzhou, China) using Illumina HiSeq 3000 platform.

Raw RNA sequencing (RNA-seq) reads were filtered using Trimmomatic v0.36. The filtered reads were mapped to the human (hg38) reference genomes using HISAT v2.1 with corresponding gene annotations (GRCh38.p13) with default settings. Total counts per mapped gene were determined using featureCounts function in SubReads package v1.5.3 with default parameter. Next, counts matrix obtained from featureCounts was used as input for differential expression gene analysis with the bioconductor package DESeq2 v1.26 in Rv4.0. Gene counts more than 5 reads 
in a single sample or more than 50 total reads across all samples were retained for further analysis. Filtered counts matrix was normalized using the DESeq2 method to remove the libraryspecific artifacts. Principal component analysis was based on global transcriptome data using the build-in function prcomp in $\mathrm{R}$ software. The genes with $\log 2$ fold change $>1$ or $<-1$ and adjusted $p<0.05$ corrected for multiple testing using the Benjamini-Hochberg method were considered significant. Transcription-factor enrichment analysis and functional enrichment analysis was performed using Metascape server tool ${ }^{81}$ (https://metascape.org/gp/index.html\#/main/step1). Gene set enrichment analysis (GSEA) used the $R$ package clusterProfiler v3.18.1. Protein-protein interaction (PPI) networks of DEGs were built using STRING v11 with a confidence score threshold of 0.7 and visualized with Cytoscape v3.8.1.

Statistical analysis

Graphpad Prism 8.0 was used for statistical analysis. For intragroup direct comparisons, Student's unpaired two-tailed $t$ test was performed to analyze significant differences. For comparisons of multiple groups, one-way ANOVAs were performed.

\section{DATA AVAILABILITY}

All raw RNA-seq data used in this study have been deposited at the https://www.ncbi. nlm.nih.gov/bioproject/PRJNA741047.

\section{ACKNOWLEDGEMENTS}

We thank Dr. Lu Lu for kind gift of reagents. This work was supported by grants from the NSFC $(82172242,81873965)$, the National Key R\&D Program of China (2020YFC0842000), and the grant from the State Key Laboratory of Respiratory Disease, Guangzhou, China (SKLRD-OP-202207). The funders had no role in study design, data collection and analysis, decision to publish, or preparation of the manuscript.

\section{AUTHOR CONTRIBUTIONS}

Conceptualization: J.H.W. Data curation: M.L.W., F.L.L., X.L., X.Y.H., and H.Y.Z. Formal analysis: M.L.W. and J.H.W. Visualization: M.L.W., F.L.L., X.Y.H., and Y.H.Z. Resources: J.H. W., J. Z., J.S., Y.T.Z, X.W.C., F.L.L., L.C., G.Y.Y., and J.C. Software: Q.Y., L.C. Writing-orginal draft: M.L.W. and J.H.W. Writing-review \& editing: X.J., J.H.W. Project administration: J.H.W., Y.T.Z., and X.W.C. Supervision J.H.W. Funding acquistion: J.H.W. and Y.T.Z. All authors have read and approved the article.

\section{ADDITIONAL INFORMATION}

Supplementary information The online version contains supplementary material available at https://doi.org/10.1038/s41392-021-00849-0.

Competing interests: The authors declare no competing interests.

\section{REFERENCES}

1. O'Driscoll, M. et al. Age-specific mortality and immunity patterns of SARS-CoV-2. Nature 590, 140-145 (2021).

2. Rubin, D., Chan-Tack, K., Farley, J. \& Sherwat, A. FDA approval of remdesivir - a step in the right direction. N. Engl. J. Med 383, 2598-2600 (2020).

3. Mahase, E. Covid-19: UK becomes first country to authorise antiviral molnupiravir. BMJ 375, n2697 (2021).

4. Mahase, E. Covid-19: Molnupiravir reduces risk of hospital admission or death by $50 \%$ in patients at risk, MSD reports. BMJ 375, n2422 (2021).

5. Sneed, T. J. \& Bible, G. H. An administrative procedure for improving staff performance in an institutional setting for retarded persons. Ment. Retard 17, 92-94 (1979).

6. Marfe, G., Perna, S. \& Shukla, A. K. Effectiveness of COVID-19 vaccines and their challenges (Review). Exp. Ther. Med. 22, 1407 (2021)

7. Merad, M. \& Martin, J. C. Pathological inflammation in patients with COVID-19: a key role for monocytes and macrophages. Nat. Rev. Immunol. 20, 355-362 (2020).

8. Vabret, N. et al. Advancing scientific knowledge in times of pandemics. Nat. Rev. Immunol. 20, 338 (2020).
9. Zhou, Z. et al. Heightened innate immune responses in the respiratory tract of COVID-19 patients. Cell Host Microbe 27, 883-890 e882 (2020).

10. Wolfel, R. et al. Virological assessment of hospitalized patients with COVID-2019. Nature 581, 465-469 (2020)

11. $\mathrm{Xu}, \mathrm{Z}$. et al. Pathological findings of COVID-19 associated with acute respiratory distress syndrome. Lancet Respiratory Med. 8, 420-422 (2020).

12. Jamal, M. et al. Immune dysregulation and system pathology in COVID-19. Virulence 12, 918-936 (2021).

13. Del Valle, D. M. et al. An inflammatory cytokine signature predicts COVID-19 severity and survival. Nat. Med. 26, 1636-1643 (2020).

14. Ren, X. et al. COVID-19 immune features revealed by a large-scale single-cell transcriptome atlas. Cell 184, 1895-1913 e1819 (2021).

15. Blanco-Melo, D. et al. Imbalanced host response to SARS-CoV-2 drives development of COVID-19. Cell 181, 1036-1045 e1039 (2020).

16. Huang, C. et al. Clinical features of patients infected with 2019 novel coronavirus in Wuhan, China. Lancet 395, 497-506 (2020).

17. Zhu, Z. et al. Clinical value of immune-inflammatory parameters to assess the severity of coronavirus disease 2019. Int J. Infect. Dis. 95, 332-339 (2020).

18. Mehta, P. et al. COVID-19: consider cytokine storm syndromes and immunosuppression. Lancet 395, 1033-1034 (2020).

19. Zhang, X. et al. Viral and host factors related to the clinical outcome of COVID-19. Nature 583, 437-440 (2020).

20. Zhou, F. et al. Clinical course and risk factors for mortality of adult inpatients with COVID-19 in Wuhan, China: a retrospective cohort study. Lancet 395, 1054-1062 (2020).

21. Herold, T. et al. Elevated levels of IL- 6 and CRP predict the need for mechanical ventilation in COVID-19. J. Allergy Clin. Immunol. 146, 128-136 e124 (2020).

22. Broman, N. et al. IL- 6 and other biomarkers as predictors of severity in COVID-19. Ann. Med. 53, 410-412 (2021).

23. Chung, J. Y., Thone, M. N. \& Kwon, Y. J. COVID-19 vaccines: the status and perspectives in delivery points of view. Adv. Drug Deliv. Rev. 170, 1-25 (2021).

24. Fajgenbaum, D. C. \& June, C. H. Cytokine Storm. N. Engl. J. Med. 383, 2255-2273 (2020).

25. Xu, X. et al. Effective treatment of severe COVID-19 patients with tocilizumab. Proc. Natl Acad. Sci. USA 117, 10970-10975 (2020).

26. La Rosee, F. et al. The Janus kinase $1 / 2$ inhibitor ruxolitinib in COVID-19 with severe systemic hyperinflammation. Leukemia 34, 1805-1815 (2020).

27. Group, R. C. et al. Dexamethasone in hospitalized patients with Covid-19. N. Engl. J. Med. 384, 693-704 (2021).

28. Lammers, T. et al. Dexamethasone nanomedicines for COVID-19. Nat. Nanotechnol. 15, 622-624 (2020).

29. Group, W. H. O. R. E. A. f. C.-T. W. et al. Association between Administration of Systemic Corticosteroids and Mortality among critically III patients With COVID19: a meta-analysis. JAMA 324, 1330-1341 (2020).

30. Stone, J. H. et al. Efficacy of tocilizumab in patients hospitalized with Covid-19. N. Engl. J. Med. 383, 2333-2344 (2020).

31. Salama, C. et al. Tocilizumab in patients hospitalized with Covid-19 pneumonia. N. Engl. J. Med 384, 20-30 (2021).

32. Della-Torre, E. et al. Interleukin-6 blockade with sarilumab in severe COVID-19 pneumonia with systemic hyperinflammation: an open-label cohort study. Ann. Rheum. Dis. 79, 1277-1285 (2020).

33. Remuzzi, A. \& Remuzzi, G. COVID-19 and Italy: what next? Lancet 395, 1225-1228 (2020).

34. Singanayagam, A. et al. Corticosteroid suppression of antiviral immunity increases bacterial loads and mucus production in COPD exacerbations. Nat. Commun. 9, 2229 (2018).

35. Thomas, B. J., Porritt, R. A., Hertzog, P. J., Bardin, P. G. \& Tate, M. D. Glucocorticosteroids enhance replication of respiratory viruses: effect of adjuvant interferon. Sci. Rep. 4, 7176 (2014).

36. Deinhardt-Emmer, S. et al. SARS-CoV-2 causes severe epithelial inflammation and barrier dysfunction. J. Virol. https://doi.org/10.1128/JVI.00110-21 (2021).

37. Degauque, N., Haziot, A., Brouard, S. \& Mooney, N. Endothelial cell, myeloid, and adaptive immune responses in SARS-CoV-2 infection. FASEB J. 35, e21577 (2021).

38. Wang, P. et al. A cross-talk between epithelium and endothelium mediates human alveolar-capillary injury during SARS-CoV-2 infection. Cell Death Dis. 11, 1042 (2020).

39. Tian, S. et al. Pathological study of the 2019 novel coronavirus disease (COVID-19) through postmortem core biopsies. Mod. Pathol. 33, 1007-1014 (2020).

40. Ackermann, M. et al. Pulmonary vascular endothelialitis, thrombosis, and angiogenesis in Covid-19. N. Engl. J. Med 383, 120-128 (2020).

41. Carroll-Portillo, A., Surviladze, Z., Cambi, A., Lidke, D. S. \& Wilson, B. S. Mast cell synapses and exosomes: membrane contacts for information exchange. Front Immunol. 3, 46 (2012).

42. Abraham, S. N. \& St John, A. L. Mast cell-orchestrated immunity to pathogens. Nat. Rev. Immunol. 10, 440-452 (2010). 
43. Marshall, J. S., Portales-Cervantes, L. \& Leong, E. Mast cell responses to viruses and pathogen products. Int. J. Mol. Sci. 20 https://doi.org/10.3390/ijms20174241 (2019).

44. Ribeiro Dos Santos Miggiolaro, A. F. et al. Covid-19 cytokine storm in pulmonary tissue: anatomopathological and immunohistochemical findings. Respir. Med. Case Rep. 31, 101292 (2020).

45. Motta Junior, J. D. S. et al. Mast cells in alveolar septa of COVID-19 patients: a pathogenic pathway that may link interstitial edema to immunothrombosis. Front Immunol. 11, 574862 (2020).

46. Malone, R. W. et al. COVID-19: famotidine, histamine, mast cells, and mechanisms. Front Pharm. 12, 633680 (2021).

47. Liu, F. L. et al. Rapid generation of ACE2 humanized inbred mouse model for COVID-19 with tetraploid complementation. Natl Sci. Rev. 8, nwaa285 (2021).

48. Jiang, R. D. et al. Pathogenesis of SARS-CoV-2 in transgenic mice expressing human angiotensin-converting enzyme 2. Cell 182, 50-58 e58 (2020).

49. $\mathrm{Gu}, \mathrm{H}$. et al. Adaptation of SARS-CoV-2 in BALB/c mice for testing vaccine efficacy. Science 369, 1603-1607 (2020).

50. Aalto, Y., Forsgren, S., Franzen, L. \& Henriksson, R. Is radiation-induced degranulation of mast cells in salivary glands induced by substance P? Oral. Oncol. 34 332-339 (1998)

51. Sun, J. et al. Generation of a broadly useful model for COVID-19 pathogenesis, vaccination, and treatment. Cell 182, 734-743 e735 (2020).

52. Song, T. Z. et al. Delayed severe cytokine storm and immune cell infiltration in SARS-CoV-2-infected aged Chinese rhesus macaques. Zool. Res. 41, 503-516 (2020).

53. Zhang, Y., Ramos, B. F. \& Jakschik, B. A. Augmentation of reverse arthus reaction by mast cells in mice. J. Clin. Invest. 88, 841-846 (1991).

54. Zhou, P. et al. A pneumonia outbreak associated with a new coronavirus of probable bat origin. Nature 579, 270-273 (2020)

55. Stefanie Deinhardt-Emmer, S. B., Clio Haring, Liane Giebeler, Andreas Henke. $<$ SARS-CoV-2 Causes Severe Epithelial Inflammation and Barrier.pdf $>$. J. Virol https://doi.org/10.1128/JVI (2021).

56. Batah, S. S. \& Fabro, A. T. Pulmonary pathology of ARDS in COVID-19: a pathological review for clinicians. Respir. Med. 176, 106239 (2021).

57. Gamperl, S., Stefanzl, G., Willmann, M., Valent, P. \& Hadzijusufovic, E. In vitro effects of histamine receptor 1 antagonists on proliferation and histamine release in canine neoplastic mast cells. Vet. Med. Sci. 7, 57-68 (2021).

58. Xie, C. L. et al. Anti-allergic compounds from the deep-sea-derived actinomycete nesterenkonia flava MCCC 1K00610. Mar Drugs 15, https://doi.org/10.3390/ md15030071 (2017).

59. Wang, Y. H. et al. Desloratadine prevents compound 48/80-induced mast cell degranulation: visualization using a vital fluorescent dye technique. Allergy $\mathbf{6 0}$, 117-124 (2005)

60. Xia, Q. et al. The effect of mizolastine on expression of vascular endothelial cell growth factor, tumour necrosis factor-alpha and keratinocyte-derived chemokine in murine mast cells, compared with dexamethasone and loratadine. Clin. Exp. Dermatol. 30, 165-170 (2005).

61. Yakuo, I. et al. [Pharmacological study of ebastine, a novel histamine H1-receptor antagonist]. Nihon Yakurigaku Zasshi 103, 121-135 (1994).

62. Palikhe, N. S. et al. Association of CRTH2 gene polymorphisms with the required dose of antihistamines in patients with chronic urticaria. Pharmacogenomics 10, 375-383 (2009)

63. Rahman, M. M., Afroz, S., Arthur, S. \& Sundaram, U. Mast cell mediated regulation of small intestinal chloride malabsorption in SAMP1/YitFc mouse model of spontaneous chronic lleitis. Cells 10, https://doi.org/10.3390/cells10030697 (2021).

64. Petito, E. et al. Association of neutrophil activation, more than platelet activation, with thrombotic complications in coronavirus disease 2019. J. Infect. Dis. 223 933-944 (2021).
65. Ueland, T. et al. Distinct and early increase in circulating MMP-9 in COVID-19 patients with respiratory failure. J. Infect. 81, e41-e43 (2020).

66. Davey, A., McAuley, D. F. \& O'Kane, C. M. Matrix metalloproteinases in acute lung injury: mediators of injury and drivers of repair. Eur. Respir. J. 38, 959-970 (2011)

67. Craig, V. J., Zhang, L., Hagood, J. S. \& Owen, C. A. Matrix metalloproteinases as therapeutic targets for idiopathic pulmonary fibrosis. Am. J. Respir. Cell Mol. Biol. 53, 585-600 (2015).

68. Hekman, R. M. et al. Actionable cytopathogenic host responses of human alveolar type 2 cells to SARS-CoV-2. Mol. Cell 80, 1104-1122 e1109 (2020).

69. Singh, D. K. et al. Responses to acute infection with SARS-CoV-2 in the lungs of rhesus macaques, baboons and marmosets. Nat. Microbiol. 6, 73-86 (2021).

70. Imai, $Y$. et al. Angiotensin-converting enzyme 2 protects from severe acute lung failure. Nature 436, 112-116 (2005).

71. Company, C. et al. Contributions of ACE and mast cell chymase to endogenous angiotensin II generation and leucocyte recruitment in vivo. Cardiovasc Res. 92 48-56 (2011).

72. Rathore, A. P. et al. Dengue virus-elicited tryptase induces endothelial permeability and shock. J. Clin. Invest. 129, 4180-4193 (2019).

73. Bouhaddou, M. et al. The global phosphorylation landscape of SARS-CoV-2 infection. Cell 182, 685-712 e619 (2020).

74. Rydyznski Moderbacher, C. et al. Antigen-specific adaptive immunity to SARS CoV-2 in acute COVID-19 and associations with age and disease severity. Cell $\mathbf{1 8 3}$ 996-1012 e1019 (2020)

75. Zheng, H. Y. et al. Pro-inflammatory microenvironment and systemic accumulation of CXCR3 + cell exacerbate lung pathology of old rhesus macaques infected with SARS-CoV-2. Signal Transduct. Target Ther. 6, 328 (2021).

76. Beigel, J. H. et al. Remdesivir for the treatment of Covid-19 - final report. N. Engl. J. Med. 383, 1813-1826 (2020).

77. Wang, Y. et al. Remdesivir in adults with severe COVID-19: a randomised, double-blind, placebo-controlled, multicentre trial. Lancet 395, 1569-1578 (2020)

78. Wadaa-Allah, A. et al. Efficacy of the current investigational drugs for the treatment of COVID-19: a scoping review. Ann. Med. 53, 318-334 (2021).

79. Fujii, S. et al. Early favipiravir treatment was associated with early defervescence in non-severe COVID-19 patients. J. Infect. Chemother. https://doi.org/10.1016/j. jiac.2021.04.013 (2021).

80. Liu, Z. et al. RBD-Fc-based COVID-19 vaccine candidate induces highly potent SARS-CoV-2 neutralizing antibody response. Signal Transduct. Target Ther. 5, 282 (2020).

81. Zhou, Y. et al. Metascape provides a biologist-oriented resource for the analysis of systems-level datasets. Nat. Commun. 10, 1523 (2019).

(i) Open Access This article is licensed under a Creative Commons Attribution 4.0 International License, which permits use, sharing, adaptation, distribution and reproduction in any medium or format, as long as you give appropriate credit to the original author(s) and the source, provide a link to the Creative Commons license, and indicate if changes were made. The images or other third party material in this article are included in the article's Creative Commons license, unless indicated otherwise in a credit line to the material. If material is not included in the article's Creative Commons license and your intended use is not permitted by statutory regulation or exceeds the permitted use, you will need to obtain permission directly from the copyright holder. To view a copy of this license, visit http://creativecommons. org/licenses/by/4.0/.

(c) The Author(s) 2021 